\title{
Current operators in integrable models: A review
}

\author{
Márton Borsi ${ }^{1,2}$, Balázs Pozsgay ${ }^{1,2}$, Levente Pristyák ${ }^{2,3}$ \\ ${ }^{1}$ Department of Theoretical Physics, \\ Eötvös Loránd University Budapest \\ 2 MTA-ELTE "Momentum" Integrable Quantum Dynamics Research Group, \\ Eötvös Loránd University Budapest \\ ${ }^{3}$ Department of Theoretical Physics, \\ Budapest University of Technology and Economics
}

\begin{abstract}
We consider the current operators of one dimensional integrable models. These operators describe the flow of the conserved charges of the models, and they play a central role in Generalized Hydrodynamics. We present the key statements about the mean currents in finite volume and in the thermodynamic limit, and we review the various proofs of the exact formulas. We also present a few new results in this review. New contributions include a computation of the currents of the Heisenberg spin chains using the string hypothesis, and simplified formulas in the thermodynamic limit. We also discuss implications of our results for the asymptotic behaviour of dynamical correlation functions.
\end{abstract}

\section{Introduction}

One dimensional integrable models are special many body systems whose exact solution is possible with analytic methods. A common property of integrable models is the existence of a large number of extra conservation laws [1]. In classical models the conserved quantities are functions on the phase space, and they have vanishing Poisson bracket. In quantum mechanics the conserved charges are described by Hermitian operators that commute with each other. In this work we treat quantum mechanical models and we put a special emphasis on lattice models, in particular on spin chains.

The existence of the extra conservation laws has dramatic effects on the dynamics of these models. First of all, it can be argued that the scattering events are always elastic, there is no momentum transfer, and thus no dissipation into the low energy modes in these systems. Furthermore, the elastic $S$-matrix factorizes: it is always a product of two-body $S$-matrices, and it does not depend on the particular temporal ordering of the scattering events. These

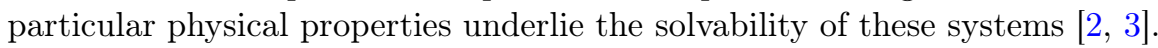

The existence of the commuting set of the charges has been known since a long time, however, many important consequences were only discovered and understood in the last 510 years. One such consequence is the absence of thermalization in integrable models: it is now understood that they equilibrate to steady states that are described by the so-called Generalized Gibbs Ensemble (GGE) 《| $\mid$ |. Furthermore, the large scale transport properties of the models can be described by focusing on the dynamics of the conserved charges only: this led to the Generalized Hydrodynamics (GHD) [5, 6], which is the topic of this special issue.

The construction of the conserved charges and the computation of their eigenvalues is well understood for an important class of integrable models: the Yang-Baxter integrable spin chains. These cases can be treated by the Quantum Inverse Scattering Method (QISM) developed by L. Faddeev and the Leningrad group [8, 8). The charges are obtained from a one-parameter family of commuting transfer matrices: they are given as expansion coefficients 
around special points. Typically the Hamiltonian is the first or second expansion coefficient and it involves nearest neighbor interactions. It is important that there are other types of integrable spin chains where the construction of the charges is much more involved: these are long range interacting models such as the Haldane-Shastry model |9] or the Inozemtsev spin chain $\mid 10]$.

In contrast to the charges, much less was known about the current operators, which describe the flow of conserved quantities under time evolution generated by the Hamiltonian. In field theories with Lorentz invariance the charge densities and the currents are intimately related: they are components of a Lorentz 2-vector. However, in spin chains and in nonrelativistic continuum models the currents are fundamentally different from the charges.

The motivation to study the current operators came mainly from GHD: the central equation of GHD for the ballistic transport is a continuity equation that involves the mean currents in a local equilibrium. It was stated in [5, 6| that the mean currents can be computed by an essentially semi-classical formula, which counts the charge eigenvalues carried by the fundamental excitations, where a semi-classical propagation speed is computed from the exact quantum mechanical solution of the models. These statements for the current mean values triggered research activity in the last 4 years, which contributed significantly to the understanding of current operators in integrable models. It is the goal of this work to review the recent results.

The paper is structured as follows. In Sec. 2 we review the key statements about the current mean values in the thermodynamic limit; these statements were first introduced in the seminal works [5, 6]. It will be the topic of the later Sections to discuss the various proofs and implications of these statements. In 3 we introduce formulas for the finite volume mean values of the currents, which are proven in later Sections. In 1 we show that the original statements of [5, 6 indeed follow from the finite volume formulas; this step has not yet been discussed in the literature. Section 5 is devoted to the current mean values of the XXX Heisenberg spin chain. Sections 6, 7 and 8 include three different proofs of the finite volume current formulas, with 7 also discussing a new algebraic construction, which embeds the current operators into the QISM framework. In Section 9 we discuss implications to the theory of factorized correlation functions. We conclude in Sec. 10 and present a list of open questions.

\section{Current mean values in the thermodynamic limit}

In this Section we explain the central statements about current operators. We do not specify the concrete integrable model, and we consider both discrete or continuous models (spin chains, and relativistic or non-relativistic field theories). The formulas for the mean values concern equilibrium situations in the thermodynamic limit, and they were introduced in the parallel papers $[5,6]$.

We consider a one dimensional integrable model defined by a Hamiltonian $H$. We assume that the model possesses a set of charges $\left\{Q_{\alpha}\right\}$ which commute with each other and $H$ is a member of the series. The charges are extensive operators and their operator density is denoted as $q_{\alpha}(x)$. Here $x$ is a space coordinate, which can be discrete (spin chains) or continuous (field theory models); the corresponding relations are

$$
Q_{\alpha}=\sum_{x} q_{\alpha}(x), \quad \text { or } \quad Q_{\alpha}=\int d x q_{\alpha}(x) .
$$

The current operators $J_{\alpha}(x)$ are defined by the continuity equation for the flow of the charge densities. We get the definitions

$$
i\left[H, q_{\alpha}(x)\right]=J_{\alpha}(x)-J_{\alpha}(x+1), \quad \text { or } \quad i\left[H, q_{\alpha}(x)\right]=-\partial_{x} J_{\alpha}(x) .
$$

Let us assume that the model can be solved by the Bethe Ansatz (the Bethe wave function will be discussed below). Then the current mean values take a rather simple form, which seems general, it does not depend on the particular details of the model. 
For simplicity let us assume that the finite volume eigenstates can be described by one set of so-called Bethe rapidities. More complicated cases with multiple types of rapidities (including the string solutions, or models solvable by the nested Bethe Ansatz) can be treated similarly. For the finite set of Bethe rapidities we will use the notation $\boldsymbol{\lambda}_{N}$, whereas in the thermodynamic limit we describe them by the root density functions $\rho(\lambda)$.

It is known that the charges act additively on the eigenstates. In the TDL the mean values of the charge densities read

$$
\left\langle q_{\alpha}(x, t)\right\rangle=\int d \lambda h_{\alpha}(\lambda) \rho(\lambda),
$$

where $h_{\alpha}(\lambda)$ is the one-particle charge eigenvalue function. For the mean values of the current operators in an equilibrium state the following formula was given in [5. 6 , 6 :

$$
\left\langle J_{\alpha}(x, t)\right\rangle=\int d \lambda v_{\mathrm{eff}}(\lambda) h_{\alpha}(\lambda) \rho(\lambda) .
$$

Here $v_{\text {eff }}(\lambda)$ is an effective velocity, which describes the propagation of a wave packet in the background of the other particles present in the system. It is a generalization of the group velocity from free models, and it is given by

$$
v_{\mathrm{eff}}(\lambda)=\frac{d e^{\mathrm{DR}}}{d p^{\mathrm{DR}}}
$$

where $e^{\mathrm{DR}}$ and $p^{\mathrm{DR}}$ are the so-called dressed energy and dressed momentum. They are defined as the energy and momentum increase as we add one more particle with rapidity $\lambda$ into the sea of particles in the equilibrium state. Precise formulas will be given later.

Equation (2.4) seems to be rather general: it is expected that it holds for every integrable model with particle conservation; the only variation between the models is the particle content, and the precise formulas for the dressed quantities. The formula is reminiscent of classical physics: the currents flowing through a point are given by the number of particles passing the point in a given time (which is given by their densities and the speed of the particles) multiplied by the charges that the particles carry. However, it turns out that the formula holds even in the quantum mechanical models, and the function $v_{\text {eff }}(\lambda)$ involves the exact solution of the quantum model. Furthermore, $v_{\text {eff }}(\lambda)$ depends on the particular physical situation, because the individual effective velocities depend on the background, which consists of all the other particles present in the system. Thus the mean currents describe the collective motion of particles, a true many body effect.

The statement (2.4) seems to hold generally in integrable models. However, as far as we know there is no general and rigorous proof of it, which would hold for every integrable model. The reason for this is simply that the different types of models require various techniques for their solutions, and there is no single universal method. However, there are a number of results available, which support or even prove (2.4) with increasing rigor and scope of validity. Let us now discuss these arguments and proofs.

It was shown in [11] that the statement always holds in models equivalent to free bosons or free fermions. In interacting cases proofs were given in various settings. The original paper |5] included a proof for relativistic QFT, and this proof was later extended in [12|. Their method is based on the so-called LeClair-Mussardo integral series [13], which describes onepoint functions in arbitrary equilibrium ensembles 14 . The statement was proven for the spin-current of the Heisenberg spin chains in [15]. In the case of the Toda chain the statement was discussed in [16]. The paper [17] includes a rather general proof based on the existence of a conserved current (a current operator which is itself a conserved charge).

All the proofs mentioned above considered the thermodynamic limit directly. An other approach was initiated in [18], where the finite volume mean values were computed in the Heisenberg spin chain. Here a new formula was found, which can be considered as the finite volume origin of (2.4); this formula will be reviewed in Section 3 below. The main result of [18] was re-derived in integrable QFT in [19]; their exact results also involves certain field theoretical correction terms which are not present in the non-relativistic setting of [18]. 
An alternative derivation for the finite volume mean values was found in 20, where a connection was found to the theory of long range deformed spin chains. Finally, in 21] a new algebraic construction was found for the current operators, which enables the computation of their mean values using standard steps of Algebraic Bethe Ansatz.

Before moving to the next Sections let us also introduce the generalized current operators $J_{\alpha, \beta}(x)$. They describe the flow of the charge $Q_{\alpha}$ under the time evolution generated by $Q_{\beta}$ [22, 18]. They are defined through the operator equation

$$
i\left[Q_{\beta}, q_{\alpha}(x)\right]=J_{\alpha, \beta}(x)-J_{\alpha, \beta}(x+1), \quad \text { or } \quad i\left[Q_{\beta}, q_{\alpha}(x)\right]=-\partial_{x} J_{\alpha, \beta}(x) .
$$

For the mean values of generalized currents the following conjecture holds:

$$
\left\langle J_{\alpha}(x, t)\right\rangle=\int d \lambda v_{\mathrm{eff}}^{\beta}(\lambda) h_{\alpha}(\lambda) \rho(\lambda),
$$

where now

$$
v_{\mathrm{eff}}^{\beta}(\lambda)=\frac{d Q_{\mathrm{dr}}^{\beta}}{d p_{\mathrm{dr}}} .
$$

This is an intermediate generalization of the effective velocity under the physical time evolution.

\section{Current mean values in finite volume}

In this Section we review the Bethe Ansatz solution of integrable models, and we present the main statements about the current mean values in finite volume.

\subsection{The Bethe Ansatz}

The Bethe Ansatz is a method invented by H. Bethe in 1931 [23] which gives the exact eigenstates of a large class of theories. The method can be applied in integrable models where particle number is conserved and where the scattering of the particles is completely elastic and factorized [3]. In such cases the exact wave function is relatively simple, and its functional form does not depend on the particular details of the model. Below we present the Bethe Ansatz solution for simple models, where the excitations do not have an inner degree of freedom. Extension to the multi-component cases is more complicated and it involves the so-called nested Bethe Ansatz, which we do not treat here.

Let us fix a reference state, which can be a ferromagnetic state in spin chains, or the Fock vacuum in field theories. We consider excitations above this vacuum. In spin chains the excitations are elementary spin waves, whereas in field theories they are the fundamental particles of the given model. In the sector with $N$ excitations let $x_{1}, x_{2}, \ldots, x_{N}$ denote the position of the particles. We require that the coordinates have a strict ordering:

$$
x_{1}<x_{2}<\cdots<x_{N}, \quad \text { or } \quad x_{1} \leq x_{2} \leq \cdots \leq x_{N} .
$$

Coinciding coordinates are forbidden in the spin- $1 / 2$ chains, where there is at most one excitation at a given site. However, coinciding coordinates are sometimes allowed in other models.

In field theories the wave functions can be extended to an arbitrary ordering using the bosonic or fermionic symmetry of the wave function, but for spin chains it is not necessary to discuss alternative orderings.

The general form of the Bethe Ansatz wave function (with the given ordering for the coordinates) is 23,8

$$
\Psi\left(x_{1}, \ldots, x_{N}\right)=\sum_{\mathcal{P} \in S_{N}}\left[\prod_{j=1}^{N} e^{i x_{j} p_{\mathcal{P}_{j}}} \prod_{\substack{j<k \\ \mathcal{P}_{j}>\mathcal{P}_{k}}} S\left(p_{j}, p_{k}\right)\right] .
$$


Here $p_{j}$ are the one-particle momenta, and $S\left(p_{j}, p_{k}\right)$ is the two-body scattering phase, and the sum runs over all permutations of the set of momenta.

The formula can be interpreted as follows. The fundamental particles move freely as long as any two of them are well separated from each other. In the sum over permutations each term corresponds to a specific spatial ordering of the particles. For each permutation $\mathcal{P} \in S_{N}$ the number $\mathcal{P}_{j}$ denotes the final position of the particle with index $j$. In the wave function there is a scattering factor $S\left(p_{j}, p_{k}\right)$ for each two-body exchange. This factor is extracted from the exact solution of the two-body problem. The wave function is sometimes called two-body irreducible, and it reflects the factorized scattering which is a general property of integrable models.

In many models it is possible to introduce the rapidity parametrization, such that the scattering phase will depend only on rapidity differences. Therefore let $\lambda$ stand for the rapidity parameter, characterizing the momentum as $p(\lambda)$, and we assume that the scattering phase is expressed as

$$
S\left(p_{j}, p_{k}\right)=S\left(p\left(\lambda_{j}\right), p\left(\lambda_{k}\right)\right)=S\left(\lambda_{j}-\lambda_{k}\right)=e^{i \delta\left(\lambda_{j}-\lambda_{k}\right)} .
$$

Putting the model into a finite volume and imposing periodic boundary conditions we obtain the Bethe equations

$$
e^{i p\left(\lambda_{j}\right)} \prod_{k \neq j} S\left(\lambda_{j}-\lambda_{k}\right)=1, \quad j=1, \ldots, N .
$$

These equations serve as magnetization conditions for the momenta of the particles. Putting them into logarithmic form we get

$$
p\left(\lambda_{j}\right)+\sum_{k \neq j} \delta\left(\lambda_{j}-\lambda_{k}\right)=2 \pi I_{j}, \quad I_{j} \in \mathbb{Z}
$$

Here $I_{j}$ are the momentum quantum numbers.

The total energy of the eigenstates is given by

$$
E=\sum_{j=1}^{N} e\left(\lambda_{j}\right)
$$

where $e(\lambda)$ is the single particle energy.

The total charge eigenvalues are

$$
Q_{\alpha}\left|\boldsymbol{\lambda}_{N}\right\rangle=\Lambda_{\alpha}\left|\boldsymbol{\lambda}_{N}\right\rangle, \quad \Lambda_{\alpha}=\sum_{j=1}^{N} h_{\alpha}\left(\lambda_{j}\right),
$$

where $h_{\alpha}(\lambda)$ is the single particle eigenvalue of the charge $Q_{\alpha}$.

The additivity of these mean values follows from the extensivity and locality of the charges, and from the mutual commutativity [3, 8].

\subsection{Mean values of the currents}

It is our goal to compute the mean currents in the finite volume Bethe states. This is a rather general problem, where the precise details of the model do not play any role. Instead, the key ingredients are the Bethe Ansatz wave function (3.2) and the Bethe equations.

First we introduce the so-called Gaudin matrix, which is given by the Jacobian of the Bethe equations

$$
G_{j k}=\frac{\partial\left(2 \pi I_{j}\right)}{\partial \lambda_{k}} .
$$

Here we treated the $I_{j}$ quantum numbers as functions of the rapidities. For further use we spell out the matrix elements:

$$
G_{j k}=\delta_{j k}\left[p^{\prime}\left(\lambda_{j}\right) L+\sum_{l} \varphi\left(\lambda_{j}-\lambda_{l}\right)\right]-\varphi\left(\lambda_{j}-\lambda_{k}\right),
$$


where we introduced the scattering kernel

$$
\varphi(\lambda)=\delta^{\prime}(\lambda)
$$

The Bethe states are distributed uniformly in the space of momentum quantum numbers $I_{j}$, thus the Gaudin determinant $\operatorname{det} G$ describes the density of states in rapidity space. Furthermore, in many models $\operatorname{det} G$ is proportional to the norm of the wave function (3.2) 24, 25, 26].

For the mean values of the currents the following exact result was found in [18]:

$$
\left\langle\boldsymbol{\lambda}_{N}\left|J_{\alpha}(x)\right| \boldsymbol{\lambda}_{N}\right\rangle=\mathbf{e}^{\prime} \cdot G^{-1} \cdot \mathbf{h}_{\alpha} .
$$

Here the quantities $\mathbf{e}^{\prime}$ and $\mathbf{h}_{\alpha}$ are $N$-dimensional vectors with elements

$$
\left(\mathbf{e}^{\prime}\right)_{j}=\frac{\partial e\left(\lambda_{j}\right)}{\partial \lambda}, \quad\left(\mathbf{h}_{\alpha}\right)_{j}=h_{\alpha}\left(\lambda_{j}\right)
$$

and $G^{-1}$ is the inverse of the Gaudin matrix.

For the generalized current operators the following result was derived in [18]:

$$
\left\langle\boldsymbol{\lambda}_{N}\left|J_{\alpha, \beta}(x)\right| \boldsymbol{\lambda}_{N}\right\rangle=\mathbf{h}_{\beta}^{\prime} \cdot G^{-1} \cdot \mathbf{h}_{\alpha} .
$$

Here $\mathbf{h}_{\beta}^{\prime}$ is an $N$-element vector with components $h_{\beta}^{\prime}\left(\lambda_{j}\right)$, and prime denotes differentiation.

These formulas can be considered a direct finite volume origin of the results (2.4). This is explained below.

\subsection{Interpretation}

The formula (3.11) has a simple semi-classical interpretation. Let us write it as

$$
\left\langle\boldsymbol{\lambda}_{N}\left|J_{\alpha}(x)\right| \boldsymbol{\lambda}_{N}\right\rangle=\frac{1}{L} \sum_{j=1}^{N} v_{\mathrm{eff}}\left(\lambda_{j}\right) h_{\alpha}\left(\lambda_{j}\right),
$$

where we defined the quantities $v_{\mathrm{eff}}\left(\lambda_{j}\right)$ as the elements of the co-vector

$$
\mathbf{v}_{\text {eff }}=\mathbf{e}^{\prime} \cdot G .
$$

Now we show that these quantities can be understood as effective velocities.

First of all we note the identity

$$
v_{\text {eff }}\left(\lambda_{j}\right)=\frac{L}{2 \pi} \frac{\partial E}{\partial I_{j}}
$$

where $E$ is the total energy of the state and $I_{j}$ are the momentum quantum numbers. This identity follows easily from (3.6) and (3.8).

Let us then discuss the structure of the formulas (3.15)-(3.16). In the case of free models we have $G_{j k}=\delta_{j k} p^{\prime}\left(\lambda_{j}\right) L$ and $p_{j} L=2 \pi I_{j}$ thus

$$
v_{\mathrm{eff}}\left(\lambda_{j}\right)=\frac{e^{\prime}\left(\lambda_{j}\right)}{p^{\prime}\left(\lambda_{j}\right)}=\frac{d e}{d p}\left(\lambda_{j}\right)
$$

is the group velocity of wave packets.

In the interacting case we see that the additional terms in $G$ result in corrections to the group velocity. The resulting effective velocity can be understood in a semi-classical picture, which was formulated in $\| 18$. The finite volume reasoning of $\|18\|$ is analogous to the argument in the infinite volume case given earlier in [27]. The argument goes as follows.

Consider the motion of $N$ particles in a periodic volume of length $L$. In the semi-classical picture we focus on the positions of the wave packets. As the particles move around the volume, they scatter on each other. In integrable models every multi-particle scattering event 
is a product of two-body scatterings, such that the result is independent of the order of the two-body events. In our cases each two-body scattering introduces a phase $\delta\left(\lambda_{j}-\lambda_{k}\right)$ to the wave function. It is known that such phases result in displacements of the wave packets, which are proportional to the scattering kernel $\varphi=\delta^{\prime}$. The displacements can be understood also as time delays. In a finite volume with a finite number of particles we intend to compute the average current of a specific charge flowing through a selected point. In order to do this we need to focus on the long time limit and compute the average number of how many times each particle crosses our point of observation. This number can be computed from the effective velocities, which describe the average propagation of the wave packets, taking into account the displacements that accumulate along the orbits. It was shown in 18 that a self-consistent computation for $v_{\text {eff }}$ results exactly in the formula (3.16).

The reader might argue that the semi-classical picture is problematic, because the spreading of the wave packet is completely ignored even though we are considering the large time limit in a finite volume. This objection is actually relevant, and the problem of the diffusion of the wave packet is already present in the derivation of [27]. Perhaps the rigorous methods of [28] could give a more rigorous semi-classical treatment.

In Section 4 it will be shown that in the thermodynamic limit (3.16) turns into the conjectured formula (2.5). Here we put forward a key idea that connects the finite and infinite volume computation: The correspondence between the formulas holds, because small changes in the dressed momentum and dressed energy can be traced back to small changes in the momentum quantum numbers and the overall finite volume energy, respectively:

$$
\delta e^{\mathrm{DR}}\left(\lambda_{j}\right) \sim \delta E, \quad \delta p^{\mathrm{DR}}\left(\lambda_{j}\right) \sim \delta\left(\frac{2 \pi I_{j}}{L}\right) .
$$

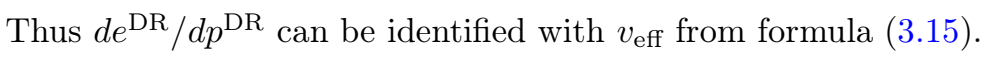

\subsection{Conjecture for more general situations}

It is tempting to formulate a general conjecture valid for a wider range of integrable models. Let us allow both lattice and continuum models, be it a relativistic or non-relativistic field theory.

Let us assume that the eigenstates of the model in question can be characterized by sets of rapidities $\left\{\boldsymbol{\lambda}_{N_{a}}^{(a)}\right\}_{a=1,2, \ldots}$. Here the index a refers to a particle species or rapidity type, and it is understood that there are $N_{a}$ rapidities for each type. Let us further assume that the eigenvalues of conserved charges can be expressed as

$$
Q_{\alpha}\left|\left\{\boldsymbol{\lambda}_{N_{a}}^{(a)}\right\}\right\rangle=\left[\sum_{a} \sum_{j=1}^{N_{a}} h_{\alpha}^{(a)}\left(\lambda_{j}^{(a)}\right)\right]\left|\left\{\boldsymbol{\lambda}_{N_{a}}^{(a)}\right\}\right\rangle,
$$

where now the functions $h_{\alpha}^{(a)}(\lambda)$ describe the one-particle eigenvalues for the particle type $a$.

Let us further assume that in finite volume the allowed sets of rapidities are given by the Bethe equations of the form

$$
p^{(a)}\left(\lambda_{j}^{(a)}\right) L+\sum_{b} \sum_{k=1}^{N_{b}} \delta^{(a, b)}\left(\lambda_{j}^{(a)}-\lambda_{k}^{(b)}\right)=2 \pi I_{j}^{(a)}+\ldots, \quad I_{j}^{(a)} \in \mathbb{Z} .
$$

Here the dots stand for correction terms, that decay exponentially with the volume, assuming that the particle number $N$ is fixed.

Then our most general conjecture for the mean values of the currents and generalized currents is

$$
\left\langle\left\{\boldsymbol{\lambda}_{N_{a}}^{(a)}\right\}\left|J_{\alpha, \beta}(x)\right|\left\{\boldsymbol{\lambda}_{N_{a}}^{(a)}\right\}\right\rangle=\mathbf{h}_{\beta}^{\prime} \cdot G^{-1} \cdot \mathbf{h}_{\alpha}+\ldots,
$$

where now $\mathbf{h}_{\alpha}$ is a vector of length $N=\sum_{a} N_{a}$ made of the one-particle eigenvalues (similarly for $\mathbf{h}_{\beta}^{\prime}$ ), and the elements of the generalized Gaudin matrix of size $N \times N$ are

$$
G_{(a, j),(b, k)}=\delta_{a b} \delta_{j k}\left[\frac{\partial p^{(a)}\left(\lambda_{j}^{(a)}\right)}{\partial \lambda} L+\sum_{c} \sum_{l} \varphi_{j l}^{(a, c)}\right]-\varphi_{j k}^{(a, b)},
$$


where

$$
\varphi_{j l}^{(a, c)}=\varphi^{(a, c)}\left(\lambda_{j}^{(a)}-\lambda_{l}^{(c)}\right), \quad \varphi^{(a, c)}=\frac{\partial \delta^{(a, c)}(\lambda)}{\partial \lambda} .
$$

Again, the dots in (3.21) stand for exponentially decaying correction terms.

There are multiple types of theories, where such a treatment is necessary. Examples include:

- Multi-component models. There is a large class of models where the fundamental excitations have an inner degree of freedom. These models can be solved by the nested Bethe Ansatz, where the different sets of rapidities correspond to the different nesting levels, describing the orientation in the internal space of the excitations. An example for such cases was considered in [20], where the current mean values of the $S U(3)$ symmetric fundamental spin chain were considered, and a result of the form (3.21) was found.

- Models with bound states. In most of the spin chains that we consider the fundamental particles can form bound states, which are described by the so-called string solutions. The bound states are stable particles, thus the different string configurations should be handled as different particle types. Energy and momentum of the string is then parametrized by the string center, and we expect that formulas of the type (3.19) should hold for the string centers. This will be treated in Section 5 below.

- Relativistic QFT. In integrable field theory there are always exponential corrections to the Bethe equations, which originate in virtual processes with virtual particles winding around the volume. We expect that (3.19) should hold in such relativistic situations, up to exponentially small corrections. For models with one particle species it was argued in 19. that this relation indeed holds.

We expect that if (3.20) and (3.19) hold exactly, then (3.21) is also exact. Otherwise there are exponentially small correction terms to the r.h.s. of (3.21).

\section{Thermodynamic limit - general treatment}

Now we perform the thermodynamic limit on the formula (3.11), focusing on simple cases where the solutions to the Bethe equations are given by real rapidities. The steps that we present here are rather standard, but for the particular problem of current mean values they were not yet published in the literature. The special case of the Heisenberg spin chain will be treated in Section 5 .

We consider a situation where in the thermodynamic limit the Bethe roots condense on the real line. In such a case the states can be described by the root densities $\rho(\lambda)$. The normalization of this function is chosen such that in a large volume $L$ the number of rapidities between $\lambda$ and $\lambda+\Delta \lambda$ is $L \rho(\lambda) \Delta \lambda$. Furthermore let us introduce the hole density $\rho_{h}(\lambda)$; a hole is a rapidity which could be a solution to the Bethe equations with the given set of particles, but which is itself not included in the set.

It follows from the Bethe equations (3.5) that

$$
\rho(\lambda)+\rho_{h}(\lambda)=\frac{1}{2 \pi} p^{\prime}(\lambda)+\int \frac{d \omega}{2 \pi} \varphi(\lambda-\omega) \rho(\omega),
$$

The equation (4.1) does not tell anything about the physical nature of the state in question; it should be regarded simply as the thermodynamic representation of the fundamental Bethe equation (3.5). On the other hand, the physical situation is specified by the filling fractions

$$
f(\lambda)=\frac{\rho(\lambda)}{\rho(\lambda)+\rho_{h}(\lambda)} .
$$

Using this definition the relation (4.1) is rewritten as

$$
\rho^{t}(\lambda)-\int \frac{d \omega}{2 \pi} \varphi(\lambda-\omega) f(\omega) \rho^{t}(\omega)=\frac{1}{2 \pi} p^{\prime}(\lambda),
$$


where we also introduced the total density

$$
\rho^{t}(\lambda)=\rho(\lambda)+\rho^{h}(\lambda) .
$$

The relation (4.3) can be written in compact notation

$$
(1-\hat{\varphi} \hat{f}) \rho^{t}=\frac{1}{2 \pi} p^{\prime}
$$

where $\hat{f}$ and $\hat{\varphi}$ are the linear operators on functions acting with multiplication with $f$ and convolution with $\varphi$, respectively.

Let us now compute the action of the Gaudin matrix in the TDL. Let $\mathbf{v}$ be a vector of length $N$ with elements given by $v\left(\lambda_{j}\right)$, where $v(\lambda)$ is a continuous function. We intend to compute the action

$$
G \mathbf{v} \text {. }
$$

First we write the matrix $G$ as

$$
G=D-\Phi,
$$

where $D$ is a diagonal matrix with elements

$$
D_{j j}=p^{\prime}\left(\lambda_{j}\right) L+\sum_{l} \varphi\left(\lambda_{j}-\lambda_{l}\right)
$$

and $\Phi_{j k}=\varphi\left(\lambda_{j}-\lambda_{k}\right)$. It follows from the construction that in the TDL

$$
D_{j j}=2 \pi L \rho^{t}(\lambda) \times\left(1+\mathcal{O}\left(L^{-1}\right)\right) .
$$

Furthermore, multiplication with the matrix $\varphi$ turns into a convolution. To be precise

$$
\sum_{k} \varphi_{j k} v\left(\lambda_{k}\right) \rightarrow L \int d \lambda \varphi\left(\lambda_{j}-\lambda\right) \rho(\lambda) v(\lambda)=L \int d \lambda \varphi\left(\lambda_{j}-\lambda\right) f(\lambda) \rho^{t}(\lambda) v(\lambda) .
$$

Altogether we find that the elements of the vector

$$
\mathbf{z}=G \mathbf{v}
$$

are given by

$$
z_{j}=2 \pi L z\left(\lambda_{j}\right) \times\left(1+\mathcal{O}\left(L^{-1}\right)\right)
$$

with

$$
z(\lambda)=\rho^{t}(\lambda) v(\lambda)-\int \frac{d \lambda^{\prime}}{2 \pi} f\left(\lambda^{\prime}\right) \rho^{t}(\lambda) \varphi\left(\lambda-\lambda^{\prime}\right) v\left(\lambda^{\prime}\right) .
$$

It is useful to introduce the "dressing equation" for $\tilde{v}(\lambda)=\rho^{t}(\lambda) v(\lambda)$, which reads

$$
z(\lambda)=\tilde{v}(\lambda)-\int \frac{d \lambda^{\prime}}{2 \pi} f\left(\lambda^{\prime}\right) \varphi\left(\lambda-\lambda^{\prime}\right) \tilde{v}\left(\lambda^{\prime}\right)
$$

This is structurally equivalent to (4.3). We call the solution of the integral equation "dressing" and denote

$$
\tilde{v}(\lambda)=z^{\mathrm{dr}}(\lambda) .
$$

Regarding the matrices we find the following relation:

$$
G D^{-1} \rightarrow(1-\hat{\varphi} \hat{f}) .
$$

It follows that for any two functions $a(\lambda), b(\lambda)$ and the corresponding vectors $\mathbf{a}$ and $\mathbf{b}$ the following holds:

$$
\begin{aligned}
\lim _{\mathrm{TDL}} \mathbf{a} G^{-1} \mathbf{b} & =a \cdot \hat{f}(1-\hat{\varphi} \hat{f})^{-1} \cdot b=a \cdot\left(\hat{f}^{-1}-\hat{\varphi}\right)^{-1} \cdot b= \\
& =\int \frac{d \lambda}{2 \pi} f(\lambda) a(\lambda) b^{\mathrm{dr}}(\lambda)=\int \frac{d \lambda}{2 \pi} f(\lambda) a^{\mathrm{dr}}(\lambda) b(\lambda) .
\end{aligned}
$$


The l.h.s. of (4.17) is symmetric with respect to $\mathbf{a}$ and $\mathbf{b}$. This symmetry is respected by the third expression, thus we can act the dressing procedure on either function.

The expression "dressing" goes back to the problem of adding/removing fundamental excitations to/from a sea of particles. The dressing operation describes the change in the charge eigenvalues in this process, and it takes into account both the charge carried by the added/removed particle, and the backflow generated by this process. Let us now discuss this connection in detail, first going back to the finite volume situation.

We consider the addition of an extra particle with rapidity $u$ to a Bethe state with rapidities $\boldsymbol{\lambda}_{N}$. The presence of the new particle will shift the rapidities of the original particles. The new rapidities will be

$$
\tilde{\lambda}_{1}, \ldots, \tilde{\lambda}_{N}, u .
$$

We assume that the shift is continuous and of order $1 / L$. Thus we write

$$
\tilde{\lambda}_{j}-\lambda_{j}=\frac{F\left(\lambda_{j} \mid u\right)}{L},
$$

where $F(\lambda \mid u)$ is the so-called shift function. It can be computed from the Bethe equations for the original particles. Expanding those equations to first order in $1 / L$ we get

$$
p^{\prime}\left(\lambda_{j}\right) F\left(\lambda_{j} \mid u\right)+\frac{1}{L} \sum_{k \neq j} \varphi\left(\lambda_{j}-\lambda_{k}\right)\left(F\left(\lambda_{j} \mid u\right)-F\left(\lambda_{k} \mid u\right)\right)+\delta\left(\lambda_{j}-u\right)=0,
$$

where we used that the momentum quantum numbers corresponding to the original particles are not changed. In the TDL this equation can be expressed as

$$
2 \pi \rho_{t}\left(\lambda_{j}\right) F\left(\lambda_{j} \mid u\right)-\int d \lambda \varphi\left(\lambda_{j}-\mu\right) \rho(\mu) F(\mu \mid u)=-\delta\left(\lambda_{j}-u\right) .
$$

Introducing

$$
2 \pi \rho_{t}(\lambda) F(\lambda \mid u)=\tilde{F}(\lambda \mid u)
$$

we get

$$
\tilde{F}(\lambda \mid u)-\int \frac{d \mu}{2 \pi} \varphi(\lambda-\mu) f(\mu) \tilde{F}(\mu \mid u)=-\frac{1}{2 \pi} \delta(\lambda-u) .
$$

We remind that the $\delta$-function here describes the scattering phase shift, and not a Diracdelta.

Let us now consider the energy difference as an effect of the addition. Calling it "dressed energy" we get the approximate finite volume expression

$$
e^{\mathrm{DR}}(u)=e(u)+\frac{1}{L} \sum_{j} e^{\prime}\left(\lambda_{j}\right) F\left(\lambda_{j} \mid u\right) .
$$

The notation ${ }^{D R}$ is not a typo here: this dressing is different from the one defined in (4.15). The connection is explained below.

In the TDL the dressed energy is expressed as

$$
e^{\mathrm{DR}}(u)=e(u)+\int \frac{d \lambda}{2 \pi} e^{\prime}(\lambda) f(\lambda) \tilde{F}(\lambda \mid u) .
$$

The equations simplify if we consider the derivative of the dressed energy with respect to the rapidity. For example we obtain

$$
\partial_{u} e^{\mathrm{DR}}(u)=\partial_{u} e(u)+\int \frac{d \lambda}{2 \pi} e^{\prime}(\lambda) f(\lambda) \partial_{u} \tilde{F}(\lambda \mid u)
$$

and for the derivative of the shift function we get

$$
\partial_{u} \tilde{F}(\lambda \mid u)-\int \frac{d \mu}{2 \pi} \varphi(\lambda-\mu) f(\mu) \partial_{u} \tilde{F}(\mu \mid u)=\frac{1}{2 \pi} \varphi(\lambda-u) .
$$


From this the following simple relation is found:

$$
\partial_{u} e^{\mathrm{DR}}(u)-\int \frac{d \mu}{2 \pi} \varphi(u-\mu) f(\mu) \partial_{\mu} e^{\mathrm{DR}}(\mu)=e^{\prime}(u) .
$$

This means that

$$
\partial_{u} e^{\mathrm{DR}}(u)=\left(e^{\prime}(u)\right)^{\mathrm{dr}}
$$

This relation connects the two different definitions for the dressing.

For the dressed momentum we obtain

$$
\partial_{u} p^{\mathrm{DR}}(u)-\int \frac{d \lambda}{2 \pi} \varphi(u-\mu) f(\mu) \partial_{\mu} p^{\mathrm{DR}}(\mu)=p^{\prime}(u) .
$$

Combining with (4.1) we get

$$
\partial_{u} p^{\mathrm{DR}}(u)=2 \pi \rho_{t}(u) .
$$

Let us now compute the current mean values. Applying the general formula (4.17) to the mean value (3.11) we get

$$
\left\langle J_{\alpha}\right\rangle=\int \frac{d \lambda}{2 \pi} f(\lambda)\left(e^{\prime}\right)^{\mathrm{dr}}(\lambda) h_{\alpha}(\lambda) .
$$

Using the definition (4.2) and (4.31) this is written as

$$
\left\langle J_{\alpha}\right\rangle=\int d \lambda \rho(\lambda) \frac{\left(e^{\prime}\right)^{\mathrm{dr}}(\lambda)}{\partial_{\lambda} p^{\mathrm{DR}}(\lambda)} h_{\alpha}(\lambda)=\int d \lambda \rho(\lambda) \frac{\partial_{\lambda} e^{\mathrm{DR}}(\lambda)}{\partial_{\lambda} p^{\mathrm{DR}}(\lambda)} h_{\alpha}(\lambda) .
$$

Making the identification (2.5) we obtain the original conjecture (2.4).

\section{Current mean values in the Heisenberg spin chain}

In this Section we treat the Heisenberg spin chains: we discuss the charges and currents of the model. The Section includes new results for the currents which were not yet presented in the literature: the discussion of the finite volume formulas in terms of the string solutions is new, together with the corresponding thermodynamic limit.

The so-called XXZ Heisenberg chain is defined by the Hamiltonian

$$
H=\sum_{j} \sigma_{j}^{x} \sigma_{j+1}^{x}+\sigma_{j}^{y} \sigma_{j+1}^{y}+\Delta\left(\sigma_{j}^{z} \sigma_{j+1}^{z}-1\right) .
$$

Here $\Delta$ is the so-called anisotropy parameter. The special point $\Delta=1$ describes the $S U(2)$ invariant XXX model, originally solved by Bethe 23). In this review we focus on the XXX case, where the fundamental particles can form bound states of arbitrary size. The XXZ model with $\Delta>1$ can be treated analogously, but the so-called massless case with $|\Delta|<1$ requires a separate treatment due to the delicate bound state structure of that model [29].

The Heisenberg chains are solved by the Bethe Ansatz. The reference state is chosen as a ferromagnetic state, for example a state with all spins up. Then the fundamental excitations are the propagating waves formed by the down spins. The exact finite volume eigenstates take the form (3.2); the characteristic functions in the XXX model are

$$
e^{i p(\lambda)}=\frac{\lambda-i / 2}{\lambda+i / 2} \quad S(\lambda)=e^{i \delta(\lambda)}=\frac{\lambda+i}{\lambda-i} .
$$

The charge and current operators in these spin chains can be obtained from the Quantum Inverse Scattering Approach. This is treated in Section 07. We just put forward that in a particular normalization the one-particle eigenvalues of the charges are

$$
\begin{aligned}
& h_{2}(\lambda)=-\frac{1}{\lambda^{2}+1 / 4} \\
& h_{\alpha}(\lambda)=\left.\partial_{x}^{\alpha-2} h_{2}(\lambda-x)\right|_{x=0} \quad \alpha>2 .
\end{aligned}
$$


Furthermore

$$
e(\lambda)=2 h_{2}(\lambda)
$$

Then mean values of the currents are given by formula (3.11). Here we do not discuss the proof of the finite volume formula, which is later presented in Sections 6 and 7 . Instead, we perform a closer analysis of the formula, with the goal of taking the proper thermodynamic limit. This consists of two steps. The first step is to take into account the bound state structure of the XXX chain: it is known that the model allows for string solutions with arbitrary length, that should be treated as separate particle types. We will show that the currents can be expressed in the form (3.21), which was put forward on general grounds. As a second step we will take the thermodynamic limit of that formula. Thereby we derive a new compact expression for the currents.

Before turning to the actual computations let us introduce generating functions for the charges and the currents. We will see that it is more convenient to work with them. For the charges we introduce the formal sum

$$
Q(\nu)=\sum_{\alpha=2}^{\infty} \frac{\nu^{\alpha-2}}{(\alpha-2) !} Q_{\alpha} .
$$

It is shown in Section 7 that this is a very natural definition which is compatible with the transfer matrix construction. This generating function is also an extensive operator, and its operator density is defined as

$$
q(\nu, x)=\sum_{\alpha=2}^{\infty} \frac{\nu^{\alpha-2}}{(\alpha-2) !} q_{\alpha}(x) .
$$

Regarding the currents we focus on the generalized operators $J_{\alpha, \beta}$ defined in (2.6). It is natural to introduce a generating function with two spectral parameters:

$$
J(\mu, \nu, x)=\sum_{\alpha=2}^{\infty} \sum_{\beta=2}^{\infty} \frac{\mu^{\alpha-2}}{(\alpha-2) !} \frac{\nu^{\beta-2}}{(\beta-2) !} J_{\alpha, \beta}(x) .
$$

This two-parameter family of operators satisfies the generalized continuity relation

$$
i[Q(\nu), q(\mu, x)]=J(\mu, \nu, x)-J(\mu, \nu, x+1) .
$$

\subsection{String solutions in finite volume}

The solutions of the Bethe equations organize themselves into the so-called strings in the complex plane. A string describes a bound state of the fundamental spin waves, where the rapidities within the string follow a well defined pattern, dictated by the pole of the $S$-matrix. In the XXX case this means that within each string the rapidities follow each other with a difference of $i$ at each step.

In the presence of multiple strings each rapidity is identified as the $r$-th element of the $\varrho$-th string of length $j$ :

$$
\lambda_{j, \varrho}^{r}=\lambda_{j, \varrho}+\frac{i}{2}(j+1-2 r)+\delta_{j, \varrho}^{r} .
$$

Here $\lambda_{j, \varrho} \in \mathbb{R}$ is the string center and $\delta_{j, \varrho}^{r}$ is the so-called string deviation which vanishes exponentially with the system size. Different string lengths correspond to different types of excitations, interpreted as bound states of elementary particles.

Considering the string structure one can reformulate the Bethe equations and the Gaudin matrix as follows 29]. Reduced equations for the string centers (also called Bethe-Takahashi equations) can be obtained by taking products of Bethe equations for the constituents within the strings. Thus we get

$$
e^{i p_{j}\left(\lambda_{j, \varrho}\right) L} \prod_{\substack{(k, \sigma) \\ \neq(j, \varrho)}} S_{j, k}\left(\lambda_{j, \varrho}-\lambda_{j, \sigma}\right)=1
$$


or in the logarithmic form

$$
L \ln \left(i p_{j}\left(\lambda_{j, \varrho}\right)\right)+\sum_{\substack{k, \sigma) \\ \neq(j, \varrho)}} \ln S_{j, k}\left(\lambda_{j, \varrho}-\lambda_{k, \sigma}\right)=2 \pi i I_{j, \varrho} .
$$

These lead to the reduced Gaudin matrix:

$$
\begin{gathered}
G_{(j, \varrho),(k, \sigma)}^{(\mathrm{r})}=\frac{\partial\left(2 \pi I_{j, \varrho}\right)}{\partial \lambda_{k, \sigma}}= \\
=\delta_{(j, \varrho),(k, \sigma)}\left(L p_{j}^{\prime}\left(\lambda_{j, \varrho}\right)+\sum_{\substack{(l, \tau) \\
\neq(j, \varrho)}} \varphi_{j, l}\left(\lambda_{j, \varrho}-\lambda_{l, \tau}\right)\right)-\left(1-\delta_{(j, \varrho),(k, \sigma)}\right) \varphi_{j, k}\left(\lambda_{j, \varrho}-\lambda_{k, \sigma}\right) .
\end{gathered}
$$

Let us define a family of functions

$$
a_{j}(\lambda)=\frac{4 j}{4 \lambda^{2}+j^{2}}, \quad j=1,2, \ldots
$$

Then the derivatives that enter the Gaudin matrix are given by

$$
p_{j}^{\prime}(\lambda)=a_{j}(\lambda)
$$

and

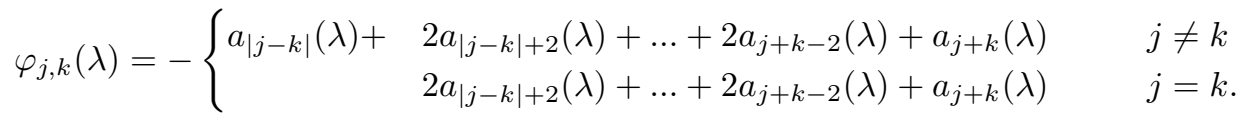

These formulas can be obtained for example by a summation of the elements of the original Gaudin matrix, corresponding to the constituents of each string:

$$
G_{(j, \varrho),(k, \sigma)}^{(\mathrm{r})}=\sum_{r=1}^{j} \sum_{s=1}^{k} G_{(j, \varrho, r),(k, \sigma, s)} .
$$

Each block of the original matrix is represented in the reduced matrix as the sum of its elements.

It was shown in 30 that the determinant of the reduced matrix describes the norm of the Bethe state with strings. Below we show that this matrix enters the formulas for the current mean values as well.

\subsection{Mean values of charges and currents with strings}

Here we compute the eigenvalues of charges and the mean values of currents using the string hypothesis. We focus on the generating functions (5.6) and (5.8) for the charges and currents, respectively.

For the charge generating function $Q(\nu)$ the single particle eigenvalues are

$$
h(\lambda \mid \nu)=-a_{1}(\lambda-\nu),
$$

where the function $a_{1}(u)$ is given by (5.15). This result can be computed from the QISM formalism, and the simple shift in $\nu$ is compatible with (5.3).

The charge eigenvalue of a $j$-string with center $\lambda_{j, \varrho}$ can be computed using a telescopic sum, and we obtain

$$
h_{j}\left(\lambda_{j, \varrho} \mid \nu\right)=\sum_{r=1}^{j} h\left(\lambda_{j, \varrho}^{r} \mid \nu\right)=-a_{j}\left(\lambda_{j, \varrho}-\nu\right) .
$$

The current mean values can also be calculated with respect to the string centers up to exponential corrections:

$$
\left\langle\left\{\lambda_{j, \varrho}\right\}_{j, \varrho}|J(\mu, \nu)|\left\{\lambda_{j, \varrho}\right\}_{j, \varrho}\right\rangle=\sum_{(j, \varrho)} \sum_{(k, \sigma)} h_{j}^{\prime}\left(\lambda_{j \varrho} \mid \nu\right) G_{(j, \varrho),(k, \sigma)}^{(\mathrm{r})^{-1}} h_{k}\left(\lambda_{k, \sigma} \mid \mu\right)+\mathcal{O}(\delta) .
$$


This can be proven starting from the finite volume formula (3.11) by finding a relation between the reduced and the ordinary inverse Gaudin matrices. Now we sketch the key steps of the proof, which is presented with all details in Appendix A.

For a complex invertible square matrix $A$ its minor corresponding to the $j$-th row and $k$-th column is defined as

$$
M_{j k}=\operatorname{det}\left(\left(A_{l m}\right)_{l \neq j, m \neq k}\right),
$$

that is, the determinant of the matrix missing the given row and column. The corresponding co-factor is

$$
C_{j k}=(-1)^{j+k} M_{j k} .
$$

Using the co-factor matrix one can express the inverse of $A$ as

$$
A^{-1}=\frac{1}{\operatorname{det} A} \cdot C^{T} .
$$

Considering the string structure the Gaudin matrix has the explicit form

$$
\begin{gathered}
G_{(j, \varrho, r),(k, \sigma, s)}=\delta_{(j, \varrho, r),(k, \sigma, s)}\left(\frac{4 L}{4 \lambda_{j, \varrho}^{r}+1}-\sum_{\begin{array}{c}
(l, \tau, t) \\
\neq(j, \varrho, r)
\end{array}} \frac{2}{\left(\lambda_{j, \varrho}^{r}-\lambda_{l, \tau}^{t}\right)^{2}+1}\right)+ \\
+\left(1-\delta_{(j, \varrho, r),(k, \sigma, s)}\right) \cdot \frac{2}{\left(\lambda_{j, \varrho}^{r}-\lambda_{k, \sigma}^{s}\right)^{2}+1} .
\end{gathered}
$$

Since adjacent rapidities in a string differ by a complex unit up to exponentially vanishing corrections, the following divergent expressions appear in the matrix:

$$
K_{j, \varrho}^{r, r+1}:=-\frac{2}{\left(\lambda_{j, \varrho}^{r+1}-\lambda_{j, \varrho}^{r}\right)^{2}+1} .
$$

Our goal is to find the leading order behaviour of the determinant and co-factors in terms of these expressions. They are present in, right above and right below the diagonal.

To calculate the Gaudin determinant the key step is to add certain rows and columns, in order to reduce the number of divergent elements in the matrix. In fact, it is possible to obtain a form when they are only present in the diagonal. This treatment is also applicable to the co-factors with a few modifications and additional row and column exchanges. The latter introduces nontrivial signs but in the end we have all divergent elements in the diagonal again.

After these simplifications the divergent expressions can be factored out from the diagonal, and we can always neglect the lower order terms in the thermodynamic limit. The remaining task is to relate the rest to the reduced matrix. This is possible because our elimination process for the divergent expressions earlier had the side-effect of summing up all elements in every block. One can show that the remaining factor is exactly the determinant of these block sums, that is, the determinant of the reduced matrix. The same is true for the co-factors as well but in that case the signs must also be matched which requires a careful analysis.

Nevertheless, the following results can be obtained:

$$
\begin{aligned}
\operatorname{det} G & =\left(\prod_{(j, \varrho} \prod_{r=1}^{j-1} K_{j, \varrho}^{r, r+1}\right) \cdot \operatorname{det} G^{(\mathrm{r})} \cdot(1+\mathcal{O}(1 / K)) \\
C_{(j, \varrho, r),(k, \sigma, s)} & =\left(\prod_{(j, \varrho)} \prod_{r=1}^{j-1} K_{j, \varrho}^{r, r+1}\right) \cdot C_{(j, \varrho),(k, \sigma)}^{(\mathrm{r})} \cdot(1+\mathcal{O}(1 / K)) \quad \forall r, s
\end{aligned}
$$

where $\mathcal{O}(1 / K)$ denotes terms vanishing exponentially. For the inverse we accordingly have

$$
G_{(j, \varrho, r),(k, \sigma, s)}^{-1}=G_{(j, \varrho),(k, \sigma)}^{(\mathrm{r})^{-1}} \cdot(1+\mathcal{O}(1 / K)) \quad \forall r, s .
$$

Interestingly, to leading order all elements are identical in a given block of the inverse matrix. From here the formula (5.21) follows immediately. The details of this computation are presented in Appendix A. 


\subsection{Thermodynamic limit: XXX chain}

In the thermodynamic limit the string centers become dense for all string types, and they will be described by density functions $\rho_{j}(\lambda)$ for particles and $\rho_{j}^{h}(\lambda)$ for holes. The Bethe equations are transformed into

$$
\rho_{j}(\lambda)+\rho_{j}^{h}(\lambda)=\frac{1}{2 \pi} p_{j}^{\prime}(\lambda)+\sum_{k=1}^{\infty} \int_{-\infty}^{\infty} \frac{d \omega}{2 \pi} \varphi_{j, k}(\lambda-\omega) \rho_{k}(\omega) .
$$

Once again we define the total root densities $\rho_{j}^{t}(\lambda)$ and the filling fractions $f_{j}(\lambda)$ so that we have the alternative form of the equations

$$
\rho_{j}^{t}(\lambda)-\sum_{k=1}^{\infty} \int_{-\infty}^{\infty} \frac{d \omega}{2 \pi} \varphi_{j, k}(\lambda-\omega) f_{k}(\omega) \rho_{j}^{t}(\omega)=\frac{1}{2 \pi} p_{j}^{\prime}(\lambda) .
$$

Using the previous compact notation now we should write

$$
(1-\underline{\underline{\hat{\varphi}} \hat{f}}) \underline{\rho}^{t}=\frac{1}{2 \pi} \underline{\underline{p}}^{\prime}
$$

where the infinite number of vector and matrix components are labeled by the string type index.

Let us examine the effect of the reduced Gaudin matrix in a similar fashion as in the real rapidity case. Consider it acting on a vector of some spectral function $v_{k, \sigma}=v_{k}\left(\lambda_{k, \sigma}\right)$ :

$$
z_{j, \varrho}:=\sum_{(k, \sigma)} G_{(j, \varrho),(k, \sigma)}^{(\mathrm{r})} v_{k, \sigma} .
$$

The main difference brought by the strings is that now our linear operator in the thermodynamic limit acts on a family of functions $\left\{v_{j}\right\}_{j}$ labeled by the string index:

$$
z_{j}(\lambda)=2 \pi L\left(\rho_{j}^{t}(\lambda) v_{j}(\lambda)-\sum_{k=1}^{\infty} \int_{-\infty}^{\infty} \frac{d \omega}{2 \pi} \varphi_{j, k}(\lambda-\omega) f_{k}(\omega) \rho_{k}^{t}(\omega) v_{k}(\omega)\right) .
$$

Using which we once again have a dressing equation for the functions $x_{j}=\frac{z_{j}}{2 \pi L}$ and $x_{j}^{\mathrm{dr}}=$ $\rho_{j}^{t} v_{j}$ :

$$
x_{j}(\lambda)=x_{j}^{\mathrm{dr}}(\lambda)-\sum_{k=1}^{\infty} \int_{-\infty}^{\infty} \frac{d \omega}{2 \pi} \varphi_{j, k}(\lambda-\omega) f_{k}(\omega) x_{j}^{\mathrm{dr}}(\omega) .
$$

In our compact notation this reads:

$$
\underline{x}=(1-\underline{\underline{\hat{\varphi}} \hat{f}}) \underline{x}^{\mathrm{dr}}=:(1-\underline{\underline{\hat{T}}}) \underline{x}^{\mathrm{dr}} .
$$

According to these results for any two spectral functions $a_{j}(\lambda)$ and $b_{j}(\lambda)$ we have the thermodynamic limit

$$
\lim _{\mathrm{TDL}} \sum_{(j, \varrho)} \sum_{(k, \sigma)} a_{j}\left(\lambda_{j, \varrho}\right) G_{(j, \varrho),(k, \sigma)}^{(\mathrm{r})-1} b_{k}\left(\lambda_{k, \sigma}\right)=\sum_{j=1}^{\infty} \int_{-\infty}^{\infty} \frac{d \lambda}{2 \pi} f_{j}(\lambda) a_{j}(\lambda) b_{j}^{\mathrm{dr}}(\lambda),
$$

or using our compact notation

$$
=\underline{a} \hat{f}(1-\underline{\underline{\varphi}} \hat{f})^{-1} \underline{b},
$$

where the dot product of the two vectors also includes integration. As in the case of real rapidities, the expression is symmetric under the exchange of the spectral functions $a$ and $b$.

As a result of the string structure, all of our formulas contain an infinite sum over the string indices. However, it is known that such equations can be partially disentangled, leading to simplified formulas |29. For the sake of completeness, we give all details of the decoupling procedure in Appendix B, and below we present the main results. 
First let us write down the decoupled form of the integral equation for the root densities. This reads

$$
\rho_{j}^{t}=\frac{1}{2 \pi} \delta_{j, 1} s+s *\left(\rho_{j-1}^{h}+\rho_{j+1}^{h}\right),
$$

where the function $s$ is

$$
s(\lambda)=\frac{\pi}{\cosh \pi \lambda}
$$

and $*$ denotes the convolution

$$
(f * g)(\lambda)=\int_{-\infty}^{\infty} \frac{d \omega}{2 \pi} f(\omega) g(\lambda-\omega) .
$$

Also $\rho_{0}^{h}=0$ was defined. The term $\frac{1}{2 \pi} \delta_{j, 1} s$ is called the source term.

For the charge mean values we have

$$
\frac{\langle Q(\nu)\rangle}{L}=\sum_{j=1}^{\infty} \int_{-\infty}^{\infty} d \lambda \rho_{j}(\lambda) h_{j}(\lambda \mid \nu)=\left[s *\left(2 \pi \rho_{1}^{h}-a_{1}\right)\right](\nu) .
$$

This relation was first derived in 31 .

Let us now consider the dressing equations (5.35). These can also be disentangled yielding

$$
\begin{aligned}
h_{j}^{\mathrm{dr}}(\lambda \mid \nu)=- & \frac{1}{2 \pi} \delta_{j, 1} s(\lambda-\nu)+ \\
& +\int_{-\infty}^{\infty} \frac{d \omega}{2 \pi} s(\lambda-\omega)\left(\eta_{j-1}(\omega) h_{j-1}^{\mathrm{dr}}(\omega \mid \nu)+\eta_{j+1}(\omega) h_{j+1}^{\mathrm{dr}}(\omega \mid \nu)\right),
\end{aligned}
$$

where $\eta_{j}=\rho_{j}^{h} / \rho_{j}^{t}$. As a result the currents become

$$
\begin{gathered}
\langle J(\mu, \nu)\rangle=\sum_{j=1}^{\infty} \int_{-\infty}^{\infty} \frac{d \lambda}{2 \pi} f_{j}(\lambda) h_{j}^{\prime}(\lambda \mid \nu) h_{j}^{\mathrm{dr}}(\lambda \mid \mu)= \\
=-\partial_{\nu}\left[\int_{-\infty}^{\infty} \frac{d \omega}{2 \pi} s(\nu-\omega) \eta_{1}(\omega) h_{1}^{\mathrm{dr}}(\omega \mid \mu)+\frac{1}{2 \pi}\left(a_{1} * s\right)(\nu-\mu)\right] .
\end{gathered}
$$

Analogous formulas in the XXZ spin chain were already given in [32], in the context of factorized correlation functions. The present formula $(5.45)$ is equivalent (apart from normalization) to the results of [32 for the quantity $\Omega$ presented in Section 5 of that work. At the time of the publication of [32] it was not clear that these objects describe current mean values. The explanation for the coincidence was only found later in [21], and it is presented below in Sections 0 and 9 .

\section{Proof using form factor expansion}

Here we present the first proof of the main statement (3.11) which appeared in [18]. Here the idea was to focus on the off-diagonal matrix elements of the charge and current operators, and to approach the mean values using a careful diagonal limit. This is possible because the form factors (matrix elements of local operators) satisfy very special relations in integrable models, and the mean values are very closely related to the off-diagonal elements. Furthermore, the continuity relations yield information about these off-diagonal elements, and this can be used to construct the mean values.

In any finite volume and for any two Bethe states with the same particle numbers the continuity equation gives

$$
i\left(\sum_{j=1}^{N} e\left(\lambda_{j}\right)-e\left(\mu_{j}\right)\right)\left\langle\boldsymbol{\lambda}_{N}\left|Q_{\alpha}(x)\right| \boldsymbol{\mu}_{N}\right\rangle=\left(1-\prod_{j=1}^{N} e^{i\left(p\left(\mu_{j}\right)-p\left(\lambda_{j}\right)\right)}\right)\left\langle\boldsymbol{\lambda}_{N}\left|J_{\alpha}(x)\right| \boldsymbol{\mu}_{N}\right\rangle .
$$


This relation only holds for the on-shell states of a finite volume theory, where the roots satisfy the Bethe equations. However, the relation can be extended to the infinite volume situation, when there is no restriction on the Bethe roots.

To this order let us consider the form factors $F^{\mathcal{O}}\left(\boldsymbol{\lambda}_{N} \mid \boldsymbol{\mu}_{N}\right)$ of an arbitrary local operator $\mathcal{O}$, which are defined as the matrix elements between infinite volume scattering states. It is known that the form factors are meromorphic functions which satisfy special properties [8]. Furthermore, the relation between the normalized off-diagonal matrix elements for generic states $\left|\boldsymbol{\lambda}_{N}\right\rangle$ and $\left|\boldsymbol{\mu}_{N}\right\rangle$ is

$$
\left\langle\boldsymbol{\lambda}_{N}|\mathcal{O}| \boldsymbol{\mu}_{N}\right\rangle=\frac{F^{\mathcal{O}}\left(\boldsymbol{\lambda}_{N} \mid \boldsymbol{\mu}_{N}\right)}{\sqrt{\operatorname{det}_{N} G\left(\boldsymbol{\lambda}_{N}\right) \operatorname{det}_{N} G\left(\boldsymbol{\mu}_{N}\right)}},
$$

The two Gaudin determinants in the denominator stem from the norm of the Bethe Ansatz wave functions. This relation was introduced for integrable QFT in [33] (where it suffers additional correction terms), and for non-relativistic models it is known to hold generally [8].

Let us define the so-called symmetric evaluation of the (infinite volume) diagonal form factors of any operator as

$$
F_{s}^{\mathcal{O}}\left(\boldsymbol{\lambda}_{N}\right)=\lim _{\varepsilon \rightarrow 0} F^{\mathcal{O}}\left(\lambda_{1}+\varepsilon, \ldots, \lambda_{N}+\varepsilon \mid \lambda_{N}, \ldots, \lambda_{1}\right) .
$$

Using (6.2) we can extend relation (6.1) also to the form factors, where there is no restriction on the Bethe roots. Introducing the short-hand notations

$$
\begin{aligned}
\mathcal{Q}_{\alpha}\left(\boldsymbol{\lambda}_{N}\right) & \equiv F_{s}^{Q_{\alpha}(0)}\left(\boldsymbol{\lambda}_{N}\right) \\
\mathcal{J}_{\alpha}\left(\boldsymbol{\lambda}_{N}\right) & \equiv F_{s}^{J_{\alpha}(0)}\left(\boldsymbol{\lambda}_{N}\right)
\end{aligned}
$$

we get from (6.1)

$$
\left(\sum_{j=1}^{N} e^{\prime}\left(\lambda_{j}\right)\right) \mathcal{Q}_{\alpha}\left(\{\lambda\}_{N}\right)=\left(\sum_{j=1}^{N} p^{\prime}\left(\lambda_{j}\right)\right) \mathcal{J}_{\alpha}\left(\{\lambda\}_{N}\right) .
$$

The finite volume mean values can be computed from special limiting values of these objects.

It is also useful to define the functions $\rho_{N}\left(\lambda_{1}, \ldots, \lambda_{N}\right)$ as the $N \times N$ Gaudin determinants evaluated at the set of rapidities $\left\{\lambda_{1}, \ldots, \lambda_{N}\right\}$. In the notations we suppress the index $N$ and write simply

$$
\rho\left(\boldsymbol{\lambda}_{N}\right)=\operatorname{det} G\left(\boldsymbol{\lambda}_{N}\right) .
$$

We remind that the Gaudin determinants describe the norms of Bethe wave functions for eigenstates, ie. for sets of rapidities satisfying the Bethe equations. On the other hand, the functions $\rho\left(\boldsymbol{\lambda}_{N}\right)$ are defined for arbitrary sets of rapidities.

The finite volume mean values of local operators can be computed through the expansion

$$
\left\langle\boldsymbol{\lambda}_{N}|\mathcal{O}(0)| \boldsymbol{\lambda}_{N}\right\rangle=\frac{\sum_{\left\{\lambda^{+}\right\} \cup\left\{\lambda^{-}\right\}} F_{s}^{\mathcal{O}}\left(\left\{\lambda^{+}\right\}\right) \rho\left(\left\{\lambda^{-}\right\}\right)}{\rho\left(\boldsymbol{\lambda}_{N}\right)},
$$

where the summation runs over all partitionings of the set of the rapidities into $\left\{\lambda^{+}\right\} \cup\left\{\lambda^{-}\right\}$. The partitionings include those cases when either subset is the empty set, and in these cases it is understood that $\rho(\emptyset)=1$ and $F_{s}^{\mathcal{O}}(\emptyset)=\langle 0|\mathcal{O}| 0\rangle$ is the v.e.v. The relation (6.7) is exact when the Bethe Ansatz wave functions are exact eigenstates of the model. It was first proposed in [34] in integrable QFT, and for the integrable spin chains it was proven first in [35] and then independently in [18].

The key idea of [18] was to use the above expansion theorem twice. First it can be used to extract the symmetric form factors of the charge density operators, because the charge mean values are known, they are given by (3.7). Then relation (6.1) gives the symmetric form factors of the current operators. Finally, the expansion theorem can be used a second time to find the explicit formula for the current mean values. 
We do not reproduce this computation here, the detailed derivations are quite lengthy and cumbersome. Here we just present the key statements.

The symmetric form factors of the charge and current operators can be expressed using graph theoretical summations. The reason for this lies in the special structure of the Gaudin matrix, which enables the use of Kirchhoff's theorem (also known as the matrix-tree theorem) for the determinant and the inverse matrix.

Let us introduce the following definitions. For a graph $\Gamma$ a directed graph $\mathcal{F}$ is a directed spanning forest of $\Gamma$ if the following conditions hold:

- $\mathcal{F}$ includes all vertices of $\Gamma$.

- $\mathcal{F}$ does not include any circles.

- Each vertex has at most one incoming edge.

The nodes that do not have incoming edges are called roots. Each spanning forest can be decomposed as a union of spanning trees, which are the connected components of the forest. Each spanning tree has exactly one root.

Using these notations the symmetric form factors of the charges are

$$
\mathcal{Q}_{\alpha}\left(\boldsymbol{\lambda}_{N}\right)=\left[\sum_{j=1}^{N} p^{\prime}\left(\lambda_{j}\right)\right]\left[\sum_{j=1}^{N} q_{\alpha}\left(\lambda_{j}\right)\right] \sum_{\mathcal{T}} \prod_{l_{j k} \in \mathcal{T}} \varphi_{j k} .
$$

Here the summation runs over all directed spanning forests of the complete graph with $N$ vertices, and the product runs over the edges of the graph.

Using (6.5) the symmetric diagonal form factors of the current operators are found to be

$$
\mathcal{J}_{\alpha}\left(\boldsymbol{\lambda}_{N}\right)=\left[\sum_{j=1}^{N} e^{\prime}\left(\lambda_{j}\right)\right]\left[\sum_{j=1}^{N} q_{\alpha}\left(\lambda_{j}\right)\right] \sum_{\mathcal{T}} \prod_{l_{j k} \in \mathcal{T}} \varphi_{j k} .
$$

Supplying these expressions to the expansion theorem (6.7) one proves the main result (3.11), as shown in [18].

This proof can be regarded as the finite volume counterpart of the proofs of [5, 12]. The works 胞, 12 operated directly in the infinite volume limit, in the framework of integrable QFT, and results for the currents were obtained through the so-called LeClair-Mussardo (LM) series. As shown earlier in |14], the LM series should be regarded as the thermodynamic limit of the expansion theorem (6.7). Thus the new addition of the work [18] was simply to sum up the series (6.7) in finite volume, and furthermore to apply and prove it in the Heisenberg chains.

We remark that essentially the same techniques can also be used in the so-called nested spin chains, where the fundamental excitations have an inner degree of freedom. In the models related to the group symmetry $S U(3)$ the finite volume form factors were investigated in [36], and a formula analogous to (6.7) was also derived. It was briefly explained in 36 that the formalism could also be used to derive and prove results for the currents in nested spin chains. However, the complexity of the method grows very quickly, and other approaches are more effective in the nested cases.

\section{$7 \quad$ Algebraic construction}

In this Section we present the algebraic construction of the charge and current operators in integrable spin chains. The charges were known since a long time: they can be computed using the so-called Quantum Inverse Scattering Method (QISM) [37, 7, 8]. However, the current operators were only constructed very recently in [21].

The main idea of the QISM is to construct a commuting set of transfer matrices, which depend on an auxiliary rapidity parameter, and to use their Taylor expansion around special points to obtain the charges. Let us therefore consider a generic integrable spin chain with local spaces $\mathbb{C}^{d}$, the total Hilbert space is thus $\mathcal{H}=\otimes_{j=1}^{L} \mathbb{C}^{d}$. Let us furthermore take an 
auxiliary space $V_{a}$, which we choose for simplicity to be identical to the physical spaces. Then The monodromy matrix acting on $V_{a} \otimes \mathcal{H}$ is defined as

$$
T_{a}(\mu)=\mathcal{L}_{a, L}(\mu) \ldots \mathcal{L}_{a, 1}(\mu) .
$$

Here $\mathcal{L}_{a, j}(\mu), j=1, \ldots, L$ are the rapidity dependent Lax operators acting on the pair of spaces with indices $a$ and $j$, where $a$ stands for the auxiliary space.

The transfer matrix is obtained as the partial trace over the auxiliary space:

$$
t(\mu)=\operatorname{Tr}_{a} T_{a}(\mu) .
$$

We require that the following exchange relation holds for the local Lax operators:

$$
R_{b, a}(\nu, \mu) \mathcal{L}_{b, j}(\nu) \mathcal{L}_{a, j}(\mu)=\mathcal{L}_{a, j}(\mu) \mathcal{L}_{b, j}(\nu) R_{b, a}(\nu, \mu)
$$

with $a, b$ referring to two different auxiliary spaces. Here $R_{b, a}(\nu, \mu)$ is the so-called $R$-matrix acting on two auxiliary spaces. Consistency requires that the $R$-matrix satisfies the so-called Yang-Baxter relation:

$$
\begin{aligned}
& R_{12}\left(\lambda_{1}, \lambda_{2}\right) R_{13}\left(\lambda_{1}, \lambda_{3}\right) R_{23}\left(\lambda_{2}, \lambda_{3}\right)= \\
& =R_{23}\left(\lambda_{2}, \lambda_{3}\right) R_{13}\left(\lambda_{1}, \lambda_{3}\right) R_{12}\left(\lambda_{1}, \lambda_{2}\right) .
\end{aligned}
$$

This is a relation for operators acting on the triple tensor product $V_{1} \otimes V_{2} \otimes V_{3}$ and we assume $V_{j} \simeq \mathbb{C}^{d}$. It is understood that each $R_{j k}$ acts only on the corresponding vector spaces. The Yang-Baxter equation can be understood as a consistency equation for factorized elastic scattering, if the $R$-matrix is interpreted as a scattering matrix [3]. Examples for $R$-matrices can be found in [38, 8, 39. In many cases $R(\mu, \nu)$ is of difference form, i.e. it only depends on the combination $\mu-\nu$. However, this is not the only possibility, and we allow for a generic dependence on both rapidity parameters.

It follows from (7.4) that $\mathcal{L}_{a, j}(\mu)=R_{a, j}\left(\mu, \xi_{0}\right)$ is a solution to (7.3), where $\xi_{0}$ is some parameter of the model. In the following we use this choice and we assume simply $\xi_{0}=0$.

The fundamental exchange relations (7.3) imply that

$$
[t(\mu), t(\nu)]=0 .
$$

The Taylor expansion of the transfer matrices are used to construct the canonical charges. Let us assume that the so-called regularity and unitarity conditions hold:

$$
\begin{aligned}
R(\lambda, \lambda) & =P \\
R_{12}\left(\lambda_{1}, \lambda_{2}\right) R_{21}\left(\lambda_{2}, \lambda_{1}\right) & =1 .
\end{aligned}
$$

Here $P$ is the permutation operator and $R_{21}(u, v)=P R_{12}(u, v) P$.

Then a generating function for global charges is defined as [8, 7]

$$
Q(\nu) \equiv(-i) t^{-1}(\nu) \frac{d}{d \nu} t(\nu) .
$$

This definition agrees with the generating function defined earlier in (5.6), thus the canonical local charges $Q_{\alpha}$ are given by its expansion coefficients. The $Q_{\alpha}$ are extensive, and the density $q_{\alpha}(x)$ spans $\alpha$ sites [40|; for example $H \sim Q_{2}$. The definition (7.7) makes sense in any finite volume, but it gives the correct $Q_{\alpha}$ if the charge fits into the volume, i.e. $L>\alpha$. In the $L \rightarrow \infty$ limit the operator $Q(\nu)$ is expected to be quasi-local, at least in some neighborhood of $\nu=0$. For proofs in concrete cases see 41, 42, 43.

In practical computations the inverse of the transfer matrix can be replaced by the space reflected TM. Defining

$$
\bar{T}_{a}(\mu)=\mathcal{L}_{a, 1}(\mu) \ldots \mathcal{L}_{a, L}(\mu), \quad \bar{t}(\mu)=\operatorname{Tr}_{a} \bar{T}_{a}(\mu)
$$




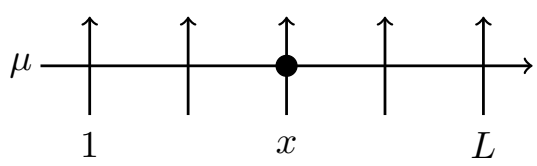

Figure 1: The generating function $q(\mu, x)$ for the charge densities is obtained by taking a derivative with respect to the rapidity at a single site. Here periodic boundary conditions are understood. The crossing with the dot denotes the action of $-i \partial_{\mu} R(\mu, 0)$.

it can be shown that the following asymptotic inversion holds:

$$
t(\lambda) \bar{t}(-\lambda)=1+\mathcal{O}\left(e^{-c L}\right),
$$

This relation holds at least in some neighborhood of $\lambda=0$, such that the decay constant $c>0$ depends on $\lambda$. For concrete proofs see $41,42,43$.

Let us now turn to the current operators. It was shown in [21] that they can be embedded into the QISM framework, which we now explain.

First we find the operator density for the generating function $Q(\mu)$. Writing $Q(\mu)=$ $\sum_{x=1}^{L} q(\mu, x)$ we can identify

$$
q(\mu, x) \equiv(-i) t^{-1}(\mu) \operatorname{Tr}_{a}\left[T_{a}^{[L, x+1]}(\mu) \partial_{\mu} \mathcal{L}_{a, x}(\mu) T_{a}^{[x-1,1]}(\mu)\right] .
$$

Here we defined the partial monodromy matrices acting on a segment $\left[x_{1} \ldots x_{2}\right]$ as

$$
T_{a}^{\left[x_{2}, x_{1}\right]}(\mu)=\mathcal{L}_{a, x_{2}}(\mu) \ldots \mathcal{L}_{a, x_{1}}(\mu) .
$$

A graphical representation of the generating function is given in Fig. 1.

We also define a generating function for the current operators. Naturally this will be a function of two auxiliary variables and a space coordinate. It is defined implicitly through the continuity relation (5.9). It is our goal to derive explicit formulas for $J(\mu, \nu, x)$.

First it can be shown using a repeated action of the Yang-Baxter equation that the solution of the analogous operator equation

$$
t^{-1}(\nu)[t(\nu), q(\mu, x)]=\Omega(\mu, \nu, x)-\Omega(\mu, \nu, x-1)
$$

is given by

$$
\Omega(\mu, \nu, x)=t^{-1}(\nu) t^{-1}(\mu) \operatorname{Tr}_{a b}\left[T_{a}^{[L, x+1]}(\mu) T_{b}^{[L, x+1]}(\nu) \Theta_{a, b}(\mu, \nu) T_{a}^{[x, 1]}(\mu) T_{b}^{[x, 1]}(\nu)\right] .
$$

where $a$ and $b$ are two different auxiliary spaces and

$$
\Theta_{a, b}(\mu, \nu)=(-i) R_{b, a}(\nu, \mu) \partial_{\mu} R_{a, b}(\mu, \nu) .
$$

is an operator insertion acting only on the auxiliary spaces. A pictorial representation of $\Omega(\mu, \nu, x)$ can be found in Fig. 2. Note that apart from the $t^{-1}$ operators $\Omega$ is a "double row" matrix product operator, and the only difference as opposed to a simple product of two transfer matrices is the insertion $\Theta_{a, b}(\mu, \nu)$, which couples two monodromy matrices.

Taking a $\nu$-derivative on the l.h.s. of (7.12) we recognize the continuity equation (5.9) and identify

$$
J(\mu, \nu, x)=-t(\nu) \partial_{\nu} \Omega(\mu, \nu, x-1) t^{-1}(\nu) .
$$

Let $|\Psi\rangle$ be an arbitrary eigenstate of the commuting transfer matrices. For the mean values we get:

$$
\langle\Psi|J(\mu, \nu, x)| \Psi\rangle=-\partial_{\nu}\langle\Psi|\Omega(\mu, \nu, x-1)| \Psi\rangle .
$$

This connects the $\nu$-derivatives of $\Omega(\mu, \nu, x)$ to the current mean values. To complete the picture, it can be shown that the initial value at $\nu=0$ is given by $\Omega(\mu, 0, x)=q(\mu, x)$. Thus $\Omega$ not only describes all (generalized) currents, but also all charge densities. 


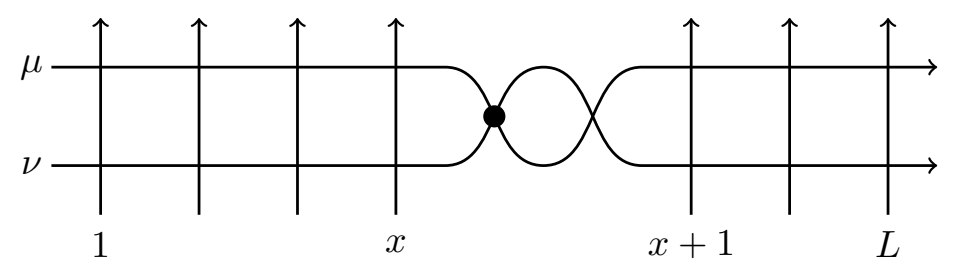

Figure 2: The operator $\Omega(\mu, \nu, x)$ positioned at site $x$. Here periodic boundary conditions are assumed in the horizontal direction. As before, the dot denotes derivative with respect to a rapidity parameter. Altogether the operator insertion in the middle is given by eq. (7.14).

It was shown in 21] that $\Omega(\mu, \nu)$ is symmetric with respect to its variables iff the $R$-matrix is of the difference form:

$$
R(\mu, \nu)=R(\mu-\nu)
$$

Examples for this are the fundamental $S U(N)$-invariant models and the XXZ and XYZ chains. A famous counter-example is the one dimensional Hubbard model.

The mean values of $\Omega(\mu, \nu)$ can be obtained with a trick that was originally developed in [44]: it can be shown that the mean values are related to a transfer matrix eigenvalue in an auxiliary problem, namely in an enlarged spin chain with two extra sites. This is a rather technical computation, and we do not discuss its details.

Let us however present the end result in the Heisenberg spin chains: it was shown in 21 that

$$
\left\langle\boldsymbol{\lambda}_{N}|\Omega(\mu, \nu)| \boldsymbol{\lambda}_{N}\right\rangle=\Psi(\mu, \nu)+\ldots,
$$

where $\Psi(\mu, \nu)$ is

$$
\Psi(\mu, \nu)=\mathbf{h}(\nu) \cdot G^{-1} \cdot \mathbf{h}(\mu)
$$

where $\mathbf{h}(\mu)$ is a vector with components given by $h_{2}\left(\lambda_{j}-\mu\right)$ with the $h_{2}$ function defined in (5.3) (similarly for $\mathbf{h}(\nu)$ ), and the dots signal correction terms that decay for small $\mu, \nu$ at least as $\mu^{L}$ or $\nu^{L}$.

Looking at the connection (7.16), and considering the expansion of $\Psi(\mu, \nu)$ we can see that the statement (3.11) is indeed reproduced by this computation. The correction terms mentioned above do not influence the final results for those generalized currents $J_{\alpha, \beta}$, which fit into a given volume, because their mean values are obtained by taking derivatives of $\Psi(\mu, \nu)$ an appropriate times at $\mu, \nu=0$.

\section{Perturbation theory and long range deformations}

A surprising new interpretation of the current operators was given in [20]: It was shown that they trigger a specific type of integrable long range deformation of spin chains, that were studied earlier in the context of the AdS/CFT correspondence [45, 46, 47]. Let us now summarize this connection.

We consider first the infinite volume situation, and perform a continuous deformation on our model, such that $\kappa$ is a deformation parameter. We require that the deformation preserves integrability, thus we look for a $\kappa$-dependent set of operators $\left\{Q_{\alpha}^{\kappa}\right\}_{\alpha=1,2, \ldots}$, such that

$$
\left[Q_{\alpha}^{\kappa}, Q_{\beta}^{\kappa}\right]=0
$$

The initial condition should be given by $Q_{\alpha}^{\kappa=0}=Q_{\alpha}$ with $Q_{\alpha}$ being the original charges. We require that the resulting operators are extensive with an operator density defined as

$$
Q_{\alpha}^{\kappa}=\sum_{x=-\infty}^{\infty} q_{\alpha}^{\kappa}(x) .
$$


It is useful to think about the deformation as a formal power series in $\kappa$, which is assumed to be convergent for the operator density at least in some neighborhood of $\kappa=0$ :

$$
Q_{\alpha}^{\kappa}=\sum_{n=0}^{\infty} \frac{\kappa^{n}}{n !} Q_{\alpha}^{(n)}
$$

Then the commutativity condition (8.1) can be analyzed order by order in $\kappa$.

The locality properties of the charges typically change under the deformation: the charge densities acquire contributions with increasing range. We can expect that the resulting charges are still quasi-local (for definitions and properties see [42|).

One possibility to construct such long range deformations is by postulating a generating equation for the charges, that describes "evolution in $\kappa$ ". This takes the Lax form 44]

$$
\frac{d}{d \kappa} Q_{\alpha}^{\kappa}=i\left[X(\kappa), Q_{\alpha}^{\kappa}\right]
$$

Here $X(\kappa)$ is a formal operator, which is chosen in a specific way such that the solution of (8.4) is a quasi-local operator. The generating equation can be understood as an iterative equation once we expand it into a Taylor series in $\kappa$.

Any such deformation leaves the commutation relations between the charges unmodified, which follows from the formal Jacobi identity:

$$
\frac{d}{d \kappa}\left[Q_{\alpha}^{\kappa}, Q_{\beta}^{\kappa}\right]=i\left[X(\kappa),\left[Q_{\alpha}^{\kappa}, Q_{\beta}^{\kappa}\right]\right] .
$$

Thus commutativity is preserved.

It was explained in [47] that there are essentially two different types of generating operators yielding non-trivial deformations, the so-called boost type and bi-local type generators. Let us focus on the boost type of deformations. In this case

$$
X(\kappa)=-\sum_{x} x q_{\alpha}^{\kappa}(x) .
$$

It was a surprising result of |20|, that in this case the deforming operators are actually the current operators. To see this, let us also define the deformed current densities as

$$
i\left[Q_{\beta}^{\kappa}, q_{\alpha}^{\kappa}(x)\right]=J_{\alpha, \beta}^{\kappa}(x)-J_{\alpha, \beta}^{\kappa}(x+1) .
$$

A simple rewriting of the deformation equation (8.4) using (8.6)-(8.7) gives

$$
\frac{d}{d \kappa} Q_{\beta}^{\kappa}=\sum_{x} J_{\alpha, \beta}^{\kappa}(x)
$$

This relation leads to the possibility of finding the current mean values using the deformation. To see this, let us assume that we can define the deformation also in finite volume, at least up to some fixed order in $\kappa$. Assuming further that we can also compute the eigenvalues of the charges as a function of $\kappa$, the current mean values of the original model will be given by the first order correction:

$$
\left\langle J_{\alpha, \beta}(x)\right\rangle=\left.\frac{1}{L} \frac{d \Lambda_{\beta}^{\kappa}}{d \kappa}\right|_{\kappa=0},
$$

where $\Lambda_{\beta}^{\kappa}$ is the $\kappa$-dependent eigenvalue of the deformed charge $Q_{\beta}^{\kappa}$.

The problem with this approach is that it is not known how to perform the long range deformation in finite volume. The difficulty lies in the "wrapping problem": the deformed operators acquire contributions with arbitrary length for any fixed $\kappa$, and generally it is not known how to "wrap" these operators around the finite volume. Existing approaches claim that the deformation can be performed at least up to some order in $\kappa$, as long as the relevant lower order contributions to the operators still fit into the given volume. In this case reliable results are found using the "Asymptotic Bethe Ansatz". The idea is to compute 
the deformation of eigenstates in infinite volume, to extract the propagation and scattering phases, and to use this information even in finite volume, to set up approximate Bethe Ansatz equations. We now summarize this approach.

First we return to the infinite volume and consider an eigenstate $\left|\Psi^{0}\right\rangle$ of the un-deformed theory. Then we define the deformation of the state as

$$
\frac{d}{d \kappa}\left|\Psi^{\kappa}\right\rangle=-i X(\kappa)\left|\Psi^{\kappa}\right\rangle
$$

with the initial condition $|\Psi(\kappa=0)\rangle=\left|\Psi^{0}\right\rangle$. The eigenstates are mapped to eigenstates and the charge eigenvalues $\Lambda_{\alpha}^{0}$ are not deformed:

$$
Q_{\alpha}^{\kappa}\left|\Psi^{\kappa}\right\rangle=\Lambda_{\alpha}^{0}\left|\Psi^{\kappa}\right\rangle
$$

However, it follows from the particular form (8.6) that the lattice momentum is changed according to

$$
\frac{d}{d \kappa} P(\kappa)=\Lambda_{\alpha}
$$

As it was discussed above, the eigenvalues of the charges do not change under the deformation, thus we get the simple solution

$$
P(\kappa)=P+\Lambda_{\alpha} \kappa .
$$

This is the generalization of the standard boost operation known in Lorentzian of Galilean invariant models. It holds for all eigenstates of the infinite volume system.

On one-particle states the deformation results in the modified momentum-rapidity relation

$$
p(\lambda) \rightarrow p(\lambda)+\kappa h_{\alpha}(\lambda) .
$$

The quasi-locality of the deformation implies that the multi-particle momentum of Bethe states has to be of the form

$$
P^{\kappa}=\sum_{j=1}^{N} p^{\kappa}\left(\lambda_{j}\right), \quad p^{\kappa}\left(\lambda_{j}\right)=p(\lambda)+\kappa h_{\alpha}\left(\lambda_{j}\right) .
$$

Furthermore it can be argued that the scattering phase in the Bethe Ansatz is not changed when viewed as a function of the rapidities.

Let us now put the theory into finite volume. The assumption of the asymptotic Bethe Ansatz is that up to a given order in $\kappa$ we can use the infinite volume data to write down the Bethe states and the corresponding Bethe equations. These will determine the finite volume rapidities. We get

$$
p^{\kappa}\left(\lambda_{j}\right) L+\sum_{k \neq j} \delta\left(\lambda_{j}-\lambda_{k}\right)=2 \pi I_{j}
$$

where $I_{j}$ are integer quantum numbers, which have to be kept fixed by continuity. Then the $\kappa$-dependence of the charge eigenvalues comes simply from the $\kappa$-dependence of the solution to this set of equations.

The first order correction to the rapidities is easily computed by taking derivatives of the above equations, leading to

$$
\frac{\partial \lambda_{j}}{\partial \kappa}=L G^{-1} \mathbf{h}_{\alpha}
$$

where $\mathbf{h}_{\alpha}$ is a vector with elements $h_{\alpha}\left(\lambda_{j}\right)$. Computing finally (8.9) gives

$$
\left\langle J_{\alpha, \beta}(x)\right\rangle=\sum_{j=1}^{N} h_{\beta}^{\prime}\left(\lambda_{j}\right) \frac{\partial \lambda_{j}}{\partial \kappa}=\mathbf{h}_{\beta}^{\prime} G^{-1} \mathbf{h}_{\alpha} .
$$

Thus we have obtained one more derivation of the formula (3.11).

The reader might wonder to what extent this argument can be considered rigorous. It was argued in [20] that the calculation can be trusted as long as the corresponding operators 
fit into a particular volume. However, there is one specific case where the computation can be made completely rigorous: the case when the deforming charge is chosen simply as the conserved spin operator. To be precise we choose

$$
X(\kappa)=-\sum_{x} x S^{z}(x) .
$$

This deformation generates a homogeneous twist along the chain, nevertheless preserving complete integrability to all orders in $\kappa$ [48. The deformed Bethe equations (8.16) hold exactly with

$$
p^{\kappa}(\lambda)=p(\lambda)-\kappa .
$$

The $\kappa$-derivative of the deformed Hamiltonian is the spin-current, and one obtains the corresponding mean value formula 1 . This twisting procedure underlies the proof of 15 for the spin current, which was given prior to the finite volume results of the present authors.

\subsection{Weak thermalization}

There is an interesting implication of the computations above: The current operators lead to a weak breaking of integrability.

Let us fix the index $\beta$, and truncate the power series (8.3) to the first order. Then we find that the operators

$$
Q_{\alpha}+\kappa \sum_{x} J_{\beta, \alpha}(x), \quad \alpha=2,3, \ldots
$$

commute up to order $\kappa$. For the case of $\alpha=2$ we get the deformation of the Hamiltonian, which reads

$$
H+\kappa \sum_{x} J_{\beta}(x)
$$

with $J_{\beta}(x)$ being the physical current of the charge $Q_{\beta}$. The computations above imply that this perturbation does not break integrability at the leading order.

The same statement was also found in two different settings using different methods.

The work [49] considered the problem of weak thermalization in cases when an integrable Hamiltonian is perturbed by some local operator. It was found that for small perturbations of order $\kappa$ there is thermalization on the time scale of $\kappa^{-2}$, if the perturbing operator is generic. However, for a special class of operators thermalization only happens on longer time scales. Interestingly, it was found in 49 that the current operators belong to this special class. However, this class of local operators is characterized by completely different tools, and at present it is not known how to connect the formalism of [49] to that of [20].

Finally, the recent work [50] studied the level spacing distribution in perturbed integrable models. It is known that integrable models show Poisson distribution, whereas in the nonintegrable cases we expect Wigner-Dyson distribution due to level repulsion. In the presence of integrability breaking one expects a crossover between the two distributions: from Poisson in intermediate volumes to Wigner-Dyson in the thermodynamic limit. Such a crossover was observed for generic integrability breaking in $\mid 51$. The work $\mid 50$ found that if the perturbation is triggered by one of current operators, then larger volumes and/or larger couplings are needed for the crossover. In particular, the critical coupling needed to cross over to the Wigner-Dyson statistics scales differently with the volume, signaling once again that the current operators do not break integrability at the leading order.

\section{Implications for correlation functions}

\subsection{Factorized correlation functions}

In the papers 18, 21] an interesting connection was pointed out to the theory of factorized correlation functions. This theory was developed in a series of works with contributions from

\footnotetext{
${ }^{1}$ We acknowledge collaboration on this computation with Lorenzo Piroli.
} 
many researchers $|52,53,54,55,56,57,58,59|$. The history of the theory started with the work [60] of Boos and Korepin who observed that some concrete multiple integral formulas for correlations of the XXX chain can be factorized (see also [61\|). Afterwards this was developed into a full algebraic theory, which deals with the normalized mean values of the form

$$
\langle\Psi|\mathcal{O}| \Psi\rangle,
$$

where $\mathcal{O}$ is a short range operator of the Heisenberg chain (the XXZ and XXX cases need to be treated separately), and $|\Psi\rangle$ is an eigenvector of the commuting set of transfer matrices. The works cited above only treated the ground state in finite and infinite volume, and also finite temperature cases, and the extension to arbitrary excited states was performed in [62, 63".

The main statement of the theory is that each mean value can be expressed using just a few functions. To be more precise, the mean values are expressed as combinations of the Taylor coefficients of some functions with one or two auxiliary variables. The number of functions depend on the model: In the XXZ chain it is enough to consider just two functions (commonly denoted as $\omega(\mu, \nu)$ and $\omega^{\prime}(\mu, \nu)$ ) for operators that are symmetric with respect to spin-flip. For generic operators an additional function $\varphi(\mu)$ is also needed. In the XXX case the situation is more involved: for operators that are $S U(2)$-symmetric (or for states with zero magnetization) it is enough to consider just a single function $\omega(\mu, \nu)$, whereas the full solution in the generic case is not yet known. We note that even the XYZ model was treated in 64 and it is conjectured that three functions with two variables are enough to describe all correlation functions in that model.

We now give a flavor for the results for the XXX chain. Let us consider states with zero total magnetization. Then short range $z-z$ correlations can be expressed as [59]

$$
\begin{aligned}
\left\langle 0\left|\sigma_{1}^{z} \sigma_{2}^{z}\right| 0\right\rangle= & \frac{1}{3}\left(1-\Psi_{0,0}\right) \\
\left\langle 0\left|\sigma_{1}^{z} \sigma_{3}^{z}\right| 0\right\rangle= & \frac{1}{3}\left(1-4 \Psi_{0,0}+\Psi_{1,1}-\frac{1}{2} \Psi_{2,0}\right) \\
\left\langle 0\left|\sigma_{1}^{z} \sigma_{4}^{z}\right| 0\right\rangle= & \frac{1}{108}\left(288 \Psi_{1,1}-15 \Psi_{2,2}+10 \Psi_{3,1}+2 \Psi_{0,0}\left(-162-42 \Psi_{1,1}+3 \Psi_{2,2}-\right.\right. \\
& \left.-2 \Psi_{3,1}\right)+\Psi_{2,0}\left(-156+12 \Psi_{1,1}-6 \Psi_{2,0}\right)+\Psi_{1,0}\left(84 \Psi_{1,0}-12 \Psi_{2,1}+\right. \\
& \left.\left.+4 \Psi_{3,0}\right)+36\right),
\end{aligned}
$$

where $\Psi_{\alpha, \gamma}{ }^{2}$ are Taylor coefficients of a function $\Psi(\mu, \nu)$, which is related to the above mentioned $\omega(\mu, \nu)$ in a linear way. The expansion reads

$$
\Psi_{\alpha, \gamma}=\left.\partial_{\mu}^{\alpha} \partial_{\nu}^{\gamma} \Psi(\mu, \nu)\right|_{\mu, \nu=0} .
$$

For our purposes the function $\Psi(\mu, \nu)$ is more convenient.

In [63 it was argued that the function $\Psi(\mu, \nu)$ is given for excited states by (7.19). However, we also explained that this is equal to the mean value of the currents. Thus, we find that the generalized current operators are those special operators whose mean values are linear in the co-efficients of $\Psi(\mu, \nu)$. This was not clear in the series of works [52, 53, 54, 55, 56, 57, 58, 59.

Furthermore, it is possible to give a rather direct connection between the operator $\Omega(\mu, \nu)$ introduced in Section 7 and the theory of factorized correlation functions. It was shown in [21] that if we define $\Omega(\mu, \nu)$ for an inhomogeneous chain with a formula analogous to (7.13), and then specify the parameters $\mu, \nu$ to two selected inhomogeneities, then actually we obtain a certain component of the corresponding two-site reduced density matrix. To be more precise, the following can be proven in a straightforward way:

$$
\Omega\left(\xi_{1}, \xi_{2}, x=2\right)=\Theta_{1,2}\left(\xi_{1}, \xi_{2}\right),
$$

\footnotetext{
${ }^{2}$ In this Section we do not use $\beta$ as an index in order to reserve it for the inverse temperature
} 
where now the operator $\Theta_{1,2}\left(\xi_{1}, \xi_{2}\right)$ acts on the physical spaces 1 and 2 . This means that for these special values $\Omega(\mu, \nu, x)$ becomes an ultra-local operator acting only on the first two sites. This is the direct bridge to the theory of factorized correlation functions. The result (9.6) is analogous to the "solution of the inverse problem" [65, 66|, where the monodromy matrix elements can be specialized such that they become ultra-local operators acting on single sites only.

\subsection{Asymptotic behaviour of dynamical correlations}

Here we present a new application of the results for correlation functions to GHD. We consider the asymptotic behaviour of correlation functions in a dynamical situation. These objects were considered in [67], where a rather general formula was derived based on the theory of hydrodynamic projections (for an alternative treatment see [68]). The most generic formula for the two-point functions concerns spatially inhomogeneous but slowly varying initial configurations, and their correlation functions in the long time and large distance limits.

Here we focus on a more restrictive setup, and we consider dynamical correlation functions in spatially homogeneous equilibrium states. The most general result of [67] can be restricted to such cases, which means that we get a hydrodynamic prediction for the dynamical correlations in equilibrium. This prediction involves certain dynamical amplitudes associated to the operators, and below we show that these amplitudes can be computed from the current mean values, using the general theory of factorized correlation functions. It is important that we do not prove the formulas of 67], we just merely apply them and compute the amplitudes that appear in them. We will see that the amplitudes also show a specific type of factorization. The results within this Subsection are new. We should note that the dynamical correlation functions of the spin chain are subject of active research [69, 70, 71, 72, 73], thus it is likely that the predictions presented below will be proven or disproven in specific situations.

Let us then consider an equilibrium state $|\Omega\rangle$ in the XXX Heisenberg spin chain, which is described by the Bethe root densities. We intend to compute the asymptotic limit of correlation functions

$$
\left\langle\Omega\left|O_{1}(x, t) O_{2}(0,0)\right| \Omega\right\rangle .
$$

We consider the asymptotic limit of this correlation function along a ray, i.e. we take $x, t \rightarrow \infty$ with $x / t=\xi$ fixed. In this limit the correlation function (9.7) becomes 67 :

$$
\left\langle\Omega\left|O_{1}(x, t) O_{2}(0,0)\right| \Omega\right\rangle=t^{-1} \sum_{j=1}^{\infty} \sum_{\lambda \in \Lambda_{j}(\xi)} \frac{\rho_{j}(\lambda) w_{j}(\lambda)}{\left|\left(v_{j}^{\mathrm{eff}}\right)^{\prime}(\lambda)\right|} V_{j}^{O_{1}}(\lambda) V_{j}^{O_{2}}(\lambda) .
$$

Here the summation over $j$ runs over the particle species, $\rho_{j}(\lambda)$ are the root densities, $v_{j}^{\text {eff }}(\lambda)$ are the effective velocities and $\Lambda_{j}(\xi)$ is the set of rapidities for which $v_{j}^{\text {eff }}(\lambda)=\xi$. Furthermore, $w_{j}(\lambda)$ are one more set of thermodynamic functions described below. Finally, $V_{j}^{O_{1}}(\lambda)$ and $V_{j}^{O_{2}}(\lambda)$ are certain amplitudes associated to the local operators, which can be interpreted as thermodynamical form factors.

These amplitudes can be computed using the theory of hydrodynamic projections [67, 74]. For the discussion of this theory we refer the reader to [67, 74]; here we just apply the formalism and show how the current mean values enter the operator amplitudes $V_{j}^{O_{1}}(\lambda)$ and $V_{j}^{O_{2}}(\lambda)$ for generic operators $O_{1}$ and $O_{2}$.

As discussed in [67], the operator amplitudes are computed by taking derivatives of the mean values of the operators with respect to parameters of the equilibrium ensemble. These parameters can be chosen for example as Lagrange multipliers of the GGE.

We explained above that the mean values of the XXX chain are factorized in the sense that they can be expressed as a sum of products of simple building blocks $\Psi_{\alpha, \gamma}$. We write 
this statement formally as

$$
\langle\mathcal{O}\rangle=\xi_{0}+\sum_{j=1}^{n} \xi_{j} \prod_{k=1}^{m_{j}} \Psi_{\alpha_{j, k}, \gamma_{j, k}} \quad \xi_{j} \in \mathbb{R},
$$

where $\xi_{j}$ are numerical coefficients, such as those present in (9.2)-(9.4).

The derivatives with respect to GGE parameters act on such a product by the Leibniz rule. If we define the amplitudes $V_{j}^{\Psi_{\alpha, \gamma}}$ for the building blocks then for any operator we have

$$
V_{j}^{\mathcal{O}}(\lambda)=\sum_{j=1}^{n} \xi_{j} \sum_{k=1}^{m_{j}} V_{j}^{\Psi_{\alpha_{j, k}, \gamma_{j, k}}}(\lambda) \prod_{\substack{l=1 \\ l \neq k}}^{m_{j}} \Psi_{\alpha_{j, k}, \gamma_{j, k}},
$$

which is merely the application of the Leibniz rule. The remaining task is to determine the functions $V_{j}^{\Psi_{\alpha, \gamma}}$. In Appendix $\bigoplus$ we show that they are given for every $\alpha, \gamma$ by

$$
V_{j}^{\Psi \alpha, \gamma}(\lambda)=\frac{1}{\pi} \frac{\left(h_{\alpha+1, j}^{\prime}\right)^{\mathrm{dr}}(\lambda) h_{\gamma+2, j}^{\mathrm{dr}}(\lambda)}{\rho_{j}^{t}(\lambda)} .
$$

This result is computed from our new formulas for the current mean values. Together with the algebraic part of the factorization procedure they yield the operator amplitudes for the asymptotic correlation functions.

\section{Conclusions and Outlook}

In this review we treated the current operators of integrable models. We showed that their mean values take a remarkably simple form, both in finite volume and in the thermodynamic limit.

This remarkable simplicity is tied to the two-body irreducibility of the Bethe Ansatz wave function. Despite the simplicity of the final formulas, the actual proofs require relatively large amount of work. The situation is similar to the problem of the norm of the Bethe Ansatz wave function: Even though the end result is always simple (it is given by the Gaudin determinant), the actual proofs are not straightforward.

Let us now summarize the advantages and disadvantages of the three different proofs that were discussed in this review:

- Form factor expansion. This method is rather general, it can be applied both in spin chains and in continuum models, be it a relativistic QFT |12| or a non-relativistic models such as the Lieb-Liniger model. A disadvantage is that the graph theoretical summations need a dedicated analysis, which can become overly complicated in multicomponent models.

- Algebraic construction. This seems to be the most general and most straightforward method for spin chains, and it is completely rigorous. Its applicability is much wider than the cases considered here. It gives a technical explanation as to why a simple final result can exist: because the mean value in question is tied to a transfer matrix eigenvalue in an auxiliary problem. However, the physical meaning of this relation is not clear. A disadvantage of the method is that in the present form it is limited to lattice models. An extension to continuous models is an exciting future direction.

- Long range deformations. This is perhaps the easiest method: Once the general recipe is understood, it can be applied to a large class of models with a very straightforward computation. A drawback is that the method is not rigorous, because the statements behind the asymptotic Bethe Ansatz are not rigorously established.

There are a number of open questions that deserve further study. We list some of these questions below: 
- Current mean values in the Hubbard model. The Hubbard model is one of the most important models of interacting 1D fermions. Its algebraic formulation is known, it involves the famous $R$-matrix of Shastry [39], which is not of difference form. The current operators in this model have not yet been studied, even though there are works treating various aspects of GHD in this model [75, 76, 77]. It would be desirable to prove the corresponding formulas for the current mean values.

- Currents in models without particle conservation. So far GHD was limited to integrable models with global $U(1)$-symmetry, leading to particle number conservation. However, the flow equations for the charges can be formulated even in the absence of particle conservation. One example for such a model is the XYZ spin chain, which is integrable and which belongs to the class of models treated by the QISM framework. Eigenstates in that model can be constructed using a generalized Algebraic Bethe Ansatz, which mixes sectors with different particle numbers. It would be desirable to work out the current mean values in this model, which would lead to a GHD setup without fundamental particle conservation.

- Long range deformations. The work [20 treated the long range deformations to first order in the deformation parameter $\kappa$. It is an interesting question, whether there are long range vertex models which could accommodate even higher order corrections. This could lead to an understanding of the current operators at higher orders in $\kappa$.

- Continuum models. The algebraic construction of Section 7 is worked out only for lattice models. It is known that certain continuum models can be obtained through special scaling limits of the spin chains. Such models include QFT's or the non-relativistic Lieb-Liniger model. It is natural to expect that the construction of current operators on the lattice is relevant also in the scaling limit. However, this has not yet been investigated.

- $T \bar{T}$-deformations. It was shown in the parallel works $|78,79|$ that the current operators also appear in the so-called bi-local deformations of the spin chains. These deformations can be considered as analogs of the famous $T \bar{T}$-deformation in QFT. It is an open question whether the actual $T \bar{T}$-deformation can be established on the lattice, and what role the algebraic construction of 7 can play in this.

- Correlation functions in nested systems. The current operators are very special: their mean values take a simple form even in nested spin chains, as discussed in [20]. Local correlation functions in nested models have been an object of interest lately [80, 81. It is now generally believed that factorization of correlation functions can not hold generally in models with higher rank symmetries 82 . It is an interesting open question whether factorized formulas can be obtained at least for a subset of local operators; if the answer is yes, then the current operators (and their algebraic construction) constitute a promising starting point for future studies.

We believe these questions deserve study, and hope that some of them will be indeed answered.

\section{Acknowledgments}

We are thankful to Bruno Bertini, Benjamin Doyon, Frank Göhmann, Yunfeng Jiang, Márton Kormos, Lorenzo Piroli, Herbert Spohn, Gábor Takács and Eric Vernier for useful discussions on various questions related to the material presented here.

\section{A Current mean values using strings - Finite volume}

Here we present the complete proof for the current formula (5.21) with the string solutions. As mentioned in the main text the key idea is to consider the divergent expressions 
(5.26) in the Gaudin matrix (5.25). We introduce the transformations that reduce the number of these elements without altering the determinant and the co-factors. Then we factor out the divergences and treat the remaining expression.

Let us introduce the transformations $R_{r}$ and $L_{r}$ adding the $r$-th column in a given block to the $(r+1)$-th (right) and $(r-1)$-th (left) respectively. Similarly $U_{r}$ and $D_{r}$ does the same with rows (up and down). They will be used to describe the elimination of divergent expressions.

In the case of the Gaudin determinant we use the following transformation in every $(j, \varrho)$ block:

$$
D_{1} D_{2} \ldots D_{j-1} R_{1} R_{2} \ldots R_{j-1} .
$$

After this the divergent expressions are only present in the diagonal, one in each position except the bottom-right of the blocks. These can be factored out in the leading order term (other terms do not contribute in the thermodynamic limit). What is left is the sub-determinant of bottom-right elements from all blocks. These elements were embedded in the whole matrix in such a way that no additional signs appear. Due to our transformations they contain the sum of their block meaning that we obtained exactly the reduced Gaudin determinant:

$$
\operatorname{det} G=\left(\prod_{(j, \varrho}^{j-1} \prod_{r=1}^{r, r+1} K_{j, \varrho}^{r}\right) \cdot \operatorname{det} G^{(\mathrm{r})} \cdot(1+\mathcal{O}(1 / K)) .
$$

Moving on to the co-factors we have one row and column missing from the matrix. We consider three separate cases: diagonal co-factors, off-diagonal co-factors of diagonal blocks and co-factors of off-diagonal blocks. The indices for the transformations are given with respect to the situation before deleting the elements.

In the case of diagonal co-factors the corresponding diagonal block $(j, \varrho)$ is, in general, divided into four regions from which the upper-left and the bottom-right contains divergent elements. Respectively we use the transformations:

$$
\begin{gathered}
D_{1} D_{2} \cdot \ldots \cdot D_{r-2} \cdot R_{1} R_{2} \cdot \ldots \cdot R_{r-2} \\
U_{j} U_{j-1} \cdot \ldots \cdot U_{r+2} \cdot L_{j} L_{j-1} \cdot \ldots \cdot L_{r+2} .
\end{gathered}
$$

where $r$ is the index of missing row and column inside the block. As a result divergent expressions are now present only in the diagonal of the block, one in each position (no exception now because one row and column is missing). In the other diagonal blocks we use the same transformation as for the determinant so that we can factor out the divergences again. The remaining factor is exactly the determinant of the reduced matrix missing one column and row, that is, the corresponding minor of the reduced matrix. It is embedded in the whole matrix properly again, implying no extra sign. The co-factor sign in the ordinary and reduced matrix match as well, since the block and the element were both diagonal. This means that for any diagonal co-factor we have

$$
C_{(j, \varrho, r),(j, \varrho, r)}=\left(\prod_{(j, \varrho)} \prod_{r=1}^{j-1} K_{j \varrho}^{r, r+1}\right) \cdot C_{(j, \varrho),(j, \varrho)}^{(\mathrm{r})} \cdot(1+\mathcal{O}(1 / K)) \quad \forall r .
$$

Next we treat the off-diagonal co-factors of diagonal blocks. Let the index (inside the block) be $r$ for the missing row and $s$ for the column. Due to symmetry it is enough to consider $s>r$. Three of the four regions contain divergences: the upper-left, the bottom-left and the bottom-right. The transformations respectively are the following (the order matters in the sense that the third must come last):

$$
\begin{gathered}
D_{1} D_{2} \cdot \ldots \cdot D_{r-2} \cdot R_{1} R_{2} \cdot \ldots \cdot R_{r-1} \\
U_{j} U_{j-1} \cdot \ldots \cdot U_{s+1} \cdot L_{j} L_{j-1} \cdot \ldots \cdot L_{s+2} \\
U_{s} U_{s-1} \cdot \ldots \cdot U_{r+2} \cdot R_{r} R_{r+1} \cdot \ldots \cdot R_{s-2} .
\end{gathered}
$$

The result is the same as before except that $(s-r)$ elements in the bottom-right region are present with negative sign. Nevertheless we treat the other diagonal blocks as usual and 
factor out again to obtain the corresponding minor of the reduced matrix. Regarding the co-factor signs the block is positive being diagonal while the element acquires the factor $(-1)^{s+r}$ which compensates for the bottom-right region. This implies that all co-factors in a diagonal block are identical:

$$
C_{(j, \varrho, r),(j, \varrho, s)}=\left(\prod_{(j, \varrho)} \prod_{r=1}^{j-1} K_{j \varrho}^{r, r+1}\right) \cdot C_{(j, \varrho),(j, \varrho)}^{(\mathrm{r})} \cdot(1+\mathcal{O}(1 / K)) \quad \forall r, s .
$$

Finally let us turn our attention to the off-diagonal blocks. Let us denote the block coordinates with $(\varrho, \sigma)$. The missing row ( $r$-th in block, $a$-th is matrix) and column ( $s$-th in block, $b$-th is matrix) now affects two different diagonal blocks. Due to symmetry we only consider $a<b$ and $\varrho<\sigma$. The transformation for the diagonal block lacking a row is

$$
\begin{gathered}
D_{1} D_{2} \cdot \ldots \cdot D_{r-2} \cdot R_{1} R_{2} \cdot \ldots \cdot R_{r-1} \\
U_{j} U_{j-1} \cdot \ldots \cdot U_{r+2} \cdot L_{j} L_{j-1} \cdot \ldots \cdot L_{r+1} .
\end{gathered}
$$

For the other block simply switch $D \leftrightarrow R$ and $U \leftrightarrow L$. The rest we treat by the usual method but now serious problems arise.

Between the two deleted diagonal elements (bottom-left region of the matrix) the new diagonal is now directly below the former one. The same is true for the blocks: the ones so far below the diagonal become the new diagonal ones. As a result the divergent elements and block sums are embedded in the matrix a nontrivial way. We can still factor out the divergences to obtain the reduced Gaudin determinant but the sign of the result is unclear. We also have the co-factor signs which are $(-1)^{p+q}$ for the ordinary matrix and $(-1)^{\pi+\rho}$ for the reduced one. We show that all these signs cancel each other.

As a first step we argue that all co-factors in a given block are identical. This is clear for the absolute values so we investigate the signs. Assume we know the sign for some element $(p, q)$. Now considering one of its neighbors from the same block essentially shifts the deleted row or column. But that only has the effect of also shifting the corresponding column or row containing blocks sums. This can be reversed by a column or row interchange in the determinant implying a minus sign which exactly cancels the one appearing in the co-factor due to considering a neighboring element. This verifies our statement.

Now we look for the common sign of the block, meaning that considering a well-chosen element is enough. This should be the bottom-right one which is favorable since it causes all block sums to accumulate also in the bottom-right element (their position in the $\varrho$-th block column and $\sigma$-th block row depends on the concrete element).

To achieve a proper embedding in the bottom-left region of the matrix we move the rows containing the block sums from the bottom to the top of the given block. Applying this to the block rows $\varrho+1, \varrho+2, \ldots \sigma$ places the divergent elements and the diagonal block sums to the diagonal thus produces a correct embedding. Relocating the rows is achieved by the interchange of neighboring ones inducing minus signs. For the $\tau$-th block row with $n_{\tau}$ rows this produces $(-1)^{n_{\tau}-1}$. For the total number of row exchanges we have

$$
\sum_{\tau=\varrho+1}^{\sigma}\left(n_{\tau}-1\right)=\sum_{\tau=\varrho+1}^{\sigma} n_{\tau}-(\sigma-\varrho)=(q-p)-(\sigma-\varrho) .
$$

This implies the final sign

$$
(-1)^{q+p} \cdot(-1)^{\sigma+\varrho}
$$

compensating for the co-factor sign of the ordinary and the reduced matrix respectively. Having all sign problems eliminated we have the general identity

$$
C_{(j, \varrho, r),(k, \sigma, s)}=\left(\prod_{(j, \varrho)} \prod_{r=1}^{j-1} K_{j, \varrho}^{r, r+1}\right) \cdot C_{(j, \varrho),(k, \sigma)}^{(\mathrm{r})} \cdot(1+\mathcal{O}(1 / K)) \quad \forall r, s
$$

And so the proof is complete. 


\section{B Decoupling of the strings}

Here we present the calculations that disentangle the string types in the various integral equations discussed in the main text, and derive the generating functions for the charge and current mean values.

First we complete the $a_{j}$ family (5.15) defining

$$
a_{0}(\lambda)=2 \pi \delta(\lambda),
$$

where now $\delta(\lambda)$ is the Dirac-delta.

Then we have the general convolution rule (now for $j, k \in \mathbb{N}$ )

$$
\left(a_{j} * a_{k}\right)(\lambda)=\int_{-\infty}^{\infty} \frac{d \omega}{2 \pi} a_{j}(\lambda-\omega) a_{k}(\omega)=a_{j+k}(\lambda),
$$

which can be used to prove the identities $(j>1, k \geq 1)$

$$
\begin{aligned}
a_{1} *\left(\varphi_{j-1, k}+\varphi_{j+1, k}\right)-\left(a_{0}+a_{2}\right) * \varphi_{j, k} & =a_{1}\left(\delta_{j-1, k}+\delta_{j+1, k}\right) \\
a_{1} * \varphi_{2, k}-\left(a_{0}+a_{2}\right) * \varphi_{1, k} & =a_{1} \delta_{2, k}
\end{aligned}
$$

that give our main tools for canceling infinite sums. Regarding the Fourier transforms we have

$$
\tilde{a}_{j}(p)=\int_{-\infty}^{\infty} \frac{d \lambda}{2 \pi} a_{j}(\lambda) e^{i \lambda p}=e^{-\frac{j|p|}{2}} .
$$

In the thermodynamic limit the Bethe equations become (5.31)

$$
\rho_{j}^{t}(\lambda)=\frac{1}{2 \pi} a_{j}(\lambda)+\sum_{k=1}^{\infty} \int_{-\infty}^{\infty} \frac{d \omega}{2 \pi} \varphi_{j, k}(\lambda-\omega) \rho_{k}(\omega) .
$$

Denoting the general equation by $[j]$ we take the following combinations:

$$
\begin{cases}\left(a_{0}+a_{2}\right) *[j]-a_{1} *([j-1]+[j+1]) & j>1 \\ \left(2 a_{0}+a_{2}\right) *[1]-a_{1} *[2] & j=1\end{cases}
$$

so that our convolution identities (B.3) and (B.4) can be utilized. We obtain the equations

$$
\begin{cases}\left(a_{0}+a_{2}\right) * \rho_{j}^{t}=a_{1} *\left(\rho_{j-1}^{h}+\rho_{j+1}^{h}\right) & j>1 \\ \left(a_{0}+a_{2}\right) * \rho_{1}^{t}=a_{1}+a_{1} * \rho_{2}^{h} & j=1,\end{cases}
$$

which can be simplified most conveniently in Fourier space. The inverse transformation can be performed using the identity

$$
\int_{-\infty}^{\infty} d p \frac{1}{2 \cosh \frac{p}{2}} e^{-i \lambda p}=\frac{\pi}{\cosh \pi \lambda}=: s(\lambda)
$$

leading to the final general result given by eq. (5.39) in the main text.

Applying these decoupled equations one can simplify both the charge and the current mean values as it is shown below. During our derivation we will use the identity

$$
s *\left(a_{j-1}+a_{j+1}\right)=a_{j} \quad j \geq 1
$$

which is easily checked in Fourier space.

For the charge mean value

$$
\frac{\langle Q(\nu)\rangle}{L}=\sum_{j=1}^{\infty} \int_{-\infty}^{\infty} d \lambda \rho_{j}(\lambda) h_{j}(\lambda \mid \nu)
$$


we substitute $h_{j}$ from (5.20) and express the particle density using the decoupled equations (5.39):

$$
\rho_{j}=\rho_{j}^{t}-\rho_{j}^{h}=\frac{1}{2 \pi} \delta_{j, 1} s+s *\left(\rho_{j-1}^{h}+\rho_{j+1}^{h}\right)-\rho_{j}^{h}
$$

to acquire the form

$$
\begin{aligned}
\frac{\langle Q(\nu)\rangle}{L}=- & {\left[s * a_{1}+2 \pi \rho_{1}^{h} *\left(s * a_{2}-a_{1}\right)+\right.} \\
& \left.\left.+2 \pi \sum_{j=2}^{\infty} \rho_{j}^{h} *\left(s *\left(a_{j-1}+a_{j+1}\right)-a_{j}\right)\right)\right](\nu)
\end{aligned}
$$

Applying (B.10) we see that the infinite sum cancels term-by-term while the other part gives the result (5.42).

For the currents we start from the dressing equations

$$
x_{j}=x_{j}^{\mathrm{dr}}-\sum_{k=1}^{\infty} \varphi_{j, k} *\left(f_{k} x_{k}^{\mathrm{dr}}\right)
$$

and take their combination according to (B.7). These can be simplified again by using the convolution identities (B.3) and (B.4) first then going to Fourier space. After the inverse transformation we have

$$
\begin{cases}x_{j}-s *\left(x_{j-1}+x_{j+1}\right)=x_{j}^{\mathrm{dr}}-s *\left(\eta_{j-1} x_{j-1}^{\mathrm{dr}}+\eta_{j+1} x_{j+1}^{\mathrm{dr}}\right) & j>1 \\ x_{1}-s * x_{2}=x_{1}^{\mathrm{dr}}-s *\left(\eta_{2} x_{2}^{\mathrm{dr}}\right) & j=1\end{cases}
$$

where we defined $\eta_{j}=\rho_{j}^{h} / \rho_{j}^{t}$. So far we worked with the general functions $x_{j}$ but since the current formula contains the charge eigenvalues let us now set

$$
x_{j}(\lambda):=h_{j}(\lambda \mid \nu)=-a_{j}(\lambda-\nu) .
$$

This makes the left hand side vanish for $j>1$ and creates the source term for $j=1$, according to (B.10). Thus we obtain (5.43).

Now we use this to reformulate the current formula. Substituting the string charges (5.20) we have

$$
\begin{gathered}
\langle J(\mu, \nu)\rangle=\sum_{j=1}^{\infty} \int_{-\infty}^{\infty} \frac{d \lambda}{2 \pi} f_{j}(\lambda) h_{j}^{\prime}(\lambda \mid \nu) h_{j}^{\mathrm{dr}}(\lambda \mid \mu)= \\
\quad=\partial_{\nu}\left[\sum_{j=1}^{\infty} a_{j} *\left(h_{j}^{\mathrm{dr}}(\cdot \mid \mu)-\eta_{j} h_{j}^{\mathrm{dr}}(\cdot \mid \mu)\right)\right](\nu) .
\end{gathered}
$$

Here it is understood that the convolution acts on the first variable of the functions $h_{j}^{\mathrm{dr}}$.

We substitute $h_{j}^{\mathrm{dr}}$ from (5.43), but only for the first term, and then rearrange the sum to obtain

$$
\begin{gathered}
\langle J(\mu, \nu)\rangle=\partial_{\nu}\left[-\frac{1}{2 \pi}\left(a_{1} * s\right)(\nu-\mu)+\left(a_{2} * s *\left(\eta_{1} h_{1}^{\mathrm{dr}}(\cdot \mid \mu)\right)-a_{1} *\left(\eta_{1} h_{1}^{\mathrm{dr}}(\cdot \mid \mu)\right)+\right.\right. \\
\left.\left.+\sum_{j=2}^{\infty}\left[s *\left(a_{j-1}+a_{j+1}\right)-a_{j}\right] *\left(\eta_{j} h_{j}^{\mathrm{dr}}(\cdot \mid \mu)\right)\right)(\nu)\right] .
\end{gathered}
$$

According to (B.10) the sum cancels term-by-term again while the rest gives (5.45). 


\section{Hydrodynamic projections and asymptotic correlation functions}

Here we give some details about the computation of the amplitudes describing the asymptotic behaviour of dynamical correlation functions. We do not discuss the theory of hydrodynamic projections, instead we refer the reader to 67, 74. Below we use the formalism of 67.

We start with the average of local operators in a GGE, described by some Lagrange multipliers $\beta_{\alpha}$. The hydrodynamic projection to the $\alpha$-th charge of an observable $\mathcal{O}$ is given by the derivative of the mean values with respect to the corresponding Lagrange multiplier.

One can always define suitable spectral functions $V_{j}^{\mathcal{O}}(\lambda)$ such that the projection is of the form

$$
\frac{\partial}{\partial \beta_{\alpha}}\langle\mathcal{O}\rangle=\sum_{j=1}^{\infty} \int_{-\infty}^{\infty} d \lambda \rho_{j}^{t}(\lambda)\left(\partial_{\beta_{\alpha}} f_{j}(\lambda)\right) V_{j}^{\mathcal{O}}(\lambda)
$$

As mentioned in the main text the building blocks have a simple relation to the generalized current mean values:

$$
\left\langle J_{\alpha, \gamma}\right\rangle=\frac{1}{2} \Psi_{\gamma-1, \alpha-2} .
$$

Therefore we only need the derivatives of the currents according to (C.1) and the desired amplitudes $V_{j}^{\Psi_{\alpha, \delta}}$ can be simply read off. In our derivation we use the differentiated dressing equation

$$
\partial_{\beta_{\delta}} x_{j}^{\mathrm{dr}}=\sum_{k=1}^{\infty} \varphi_{j, k} *\left(\left(\partial_{\beta_{\delta}} f_{k}\right) x_{k}^{\mathrm{dr}}+f_{k}\left(\partial_{\beta_{\delta}} x_{k}^{\mathrm{dr}}\right)\right) .
$$

We analyze a general expression with any spectral functions $\left\{x_{j}\right\}_{j=0}^{\infty}$ and $\left\{y_{j}\right\}_{j=0}^{\infty}$. For simplicity we hide the $\lambda$ dependence of all functions.

$$
\partial_{\beta_{\delta}} \int_{-\infty}^{\infty} \frac{d \lambda}{2 \pi} x_{j} f_{j} y_{j}^{\mathrm{dr}}=\int_{-\infty}^{\infty} \frac{d \lambda}{2 \pi} x_{j}\left(\left(\partial_{\beta_{\delta}} f_{j}\right) y_{j}^{\mathrm{dr}}+f_{j}\left(\partial_{\beta_{\delta}} y_{j}^{\mathrm{dr}}\right)\right) .
$$

According to C.3 we successively substitute $\partial_{\beta_{\delta}} y_{j}^{\mathrm{dr}}$ obtaining an infinite series

$$
\int_{-\infty}^{\infty} \frac{d \lambda}{2 \pi}\left(x_{j}\left(\partial_{\beta_{\delta}} f_{j}\right) y_{j}^{\mathrm{dr}}+x_{j} f_{j} \sum_{k=1}^{\infty} \varphi_{j, k} *\left(\left(\partial_{\beta_{\delta}} f_{k}\right) y_{k}^{\mathrm{dr}}\right)+\ldots\right) .
$$

Using the operator $\underline{\underline{T}}$ and the compact formalism defined in $(\sqrt{5.36})$ (now omitting the matrix and vector notations) we have

$$
\begin{aligned}
& \int_{-\infty}^{\infty} \frac{d \lambda}{2 \pi} x_{j} f_{j}\left[\left(\sum_{k=0}^{\infty} \hat{T}^{k}\right)\left(\frac{\partial_{\beta_{\delta}} f}{f} y^{\mathrm{dr}}\right)\right]_{j}= \\
& =\int_{-\infty}^{\infty} \frac{d \lambda}{2 \pi} x_{j} f_{j}\left[(1-\hat{T})^{-1}\left(\frac{\partial_{\beta_{\delta}} f}{f} y^{\mathrm{dr}}\right)\right]_{j},
\end{aligned}
$$

where the dressing operator can be recognized. We use its symmetry to obtain our final result

$$
\int_{-\infty}^{\infty} \frac{d \lambda}{2 \pi}\left(\partial_{\beta_{\delta}} f_{j}\right) x_{j}^{\mathrm{dr}} y_{j}^{\mathrm{dr}}
$$

Simple substitutions lead to the derivative of the current mean value:

$$
\partial_{\beta_{\delta}}\left\langle J_{\alpha, \gamma}\right\rangle=\sum_{j=1}^{\infty} \int_{-\infty}^{\infty} \frac{d \lambda}{2 \pi}\left(\partial_{\beta_{\delta}} f_{j}(\lambda)\right)\left(h_{\gamma, j}^{\prime}\right)^{\mathrm{dr}}(\lambda) h_{\alpha, j}^{\mathrm{dr}}(\lambda)
$$

from which the desired functions for the building blocks can be read off according to (C.1). Thus we obtain 9.11). 


\section{References}

[1] J.-S. Caux and J. Mossel, "Remarks on the notion of quantum integrability," J. Stat. Mech. 2011 (2011) 02023, arXiv:1012.3587 [cond-mat.str-el].

[2] B. Sutherland, Beautiful Models. World Scientific Publishing Company, 2004.

[3] G. Mussardo, "Off-critical statistical models: Factorized scattering theories and bootstrap program," Phys. Rept. 218 (1992) 215-379.

[4] F. H. L. Essler and M. Fagotti, "Quench dynamics and relaxation in isolated integrable quantum spin chains," J. Stat. Mech. 6 (2016) 064002, arXiv:1603.06452 [cond-mat.quant-gas].

[5] O. A. Castro-Alvaredo, B. Doyon, and T. Yoshimura, "Emergent Hydrodynamics in Integrable Quantum Systems Out of Equilibrium," Phys. Rev. X 6 (2016) no. 4. 041065, arXiv: 1605.07331 [cond-mat.stat-mech].

[6] B. Bertini, M. Collura, J. De Nardis, and M. Fagotti, "Transport in Out-of-Equilibrium XXZ Chains: Exact Profiles of Charges and Currents," Phys. Rev. Lett. 117 (2016) no. 20, 207201, arXiv:1605.09790 [cond-mat.stat-mech].

[7] L. Faddeev, "Instructive history of the quantum inverse scattering method," Actd Applicandae Mathematicae 39 (1995) 69-84.

[8] V. Korepin, N. Bogoliubov, and A. Izergin, Quantum inverse scattering method and correlation functions. Cambridge University Press, 1993.

[9] J. C. Talstra and F. D. M. Haldane, "Integrals of motion of the Haldane-Shastry model," Journal of Physics A: Mathematical and General 28 (1995) no. 8, 2369-2377, arXiv: cond-mat/9411065 [cond-mat].

[10] V. I. Inozemtsev, "On the connection between the one-dimensional S=1/2 Heisenberg chain and Haldane-Shastry model," Journal of Statistical Physics 59 (1990) no. 5. 1143-1155.

[11] M. Fagotti, "Charges and currents in quantum spin chains: late-time dynamics and spontaneous currents," J. Phys. A 50 (2017) no. 3, 034005, arXiv:1608.02869 [cond-mat.stat-mech].

[12] D.-L. Vu and T. Yoshimura, "Equations of state in generalized hydrodynamics," SciPost Phys. 6 (2019) 23, arXiv:1809.03197 [cond-mat.stat-mech].

[13] A. Leclair and G. Mussardo, "Finite temperature correlation functions in integrable QFT," Nucl. Phys. B552 (1999) 624-642, arXiv:hep-th/9902075.

[14] B. Pozsgay, "Mean values of local operators in highly excited Bethe states," J. Stat. Mech. 2011 (2011) P01011, arXiv:1009.4662 [hep-th].

[15] A. Urichuk, Y. Oez, A. Klümper, and J. Sirker, "The spin Drude weight of the XXZ chain and generalized hydrodynamics," SciPost Phys. 6 (2019) 5, arXiv:1808.09033.

[16] H. Spohn, "The collision rate ansatz for the classical Toda lattice," Phys. Rev. E 101 (2020) 060103, arXiv:2004.03802 [cond-mat.stat-mech].

[17] T. Yoshimura and H. Spohn, "Collision rate ansatz for quantum integrable systems," SciPost Phys. 9 (2020) 40, arXiv:2004.07113 [cond-mat.stat-mech].

[18] M. Borsi, B. Pozsgay, and L. Pristyák, "Current operators in Bethe Ansatz and Generalized Hydrodynamics: An exact quantum/classical correspondence," Phys. Rev. $X 10$ (2020) 011054, arXiv:1908.07320 [cond-mat.stat-mech].

[19] Z. Bajnok and I. Vona, "Exact finite volume expectation values of conserved currents," Phys. Lett. B 805 (2020) 135446, arXiv:1911.08525 [hep-th].

[20] B. Pozsgay, "Current operators in integrable spin chains: lessons from long range deformations," SciPost Phys. 8 (2020) 016, arXiv:1910.12833 [cond-mat.stat-mech]. 
[21] B. Pozsgay, "Algebraic construction of current operators in integrable spin chains," Phys. Rev. Lett. 125 (2020) no. 7, 070602, arXiv:2005.06242 [cond-mat.stat-mech].

[22] B. Doyon and T. Yoshimura, "A note on generalized hydrodynamics: inhomogeneous fields and other concepts," SciPost Phys. 2 (2017) 014, arXiv:1611.08225 [cond-mat.stat-mech].

[23] H. Bethe, "Zur Theorie der Metalle," Zeitschrift für Physik A71 (1931) 205.

[24] M. Gaudin, "La function d'onde de Bethe pour les modèles exacts de la mécanique statistique," Commisariat á l'énergie atomique, Paris (1983).

[25] M. Gaudin, B. M. McCoy, and T. T. Wu, "Normalization sum for the Bethe's hypothesis wave functions of the Heisenberg-Ising chain," Phys. Rev. D 23 (1981) 417-419.

[26] V. E. Korepin, "Calculation of norms of Bethe wave functions," Comm. Math. Phys. 86 (1982) 391.

[27] B. Doyon, T. Yoshimura, and J.-S. Caux, "Soliton Gases and Generalized Hydrodynamics," Phys. Rev. Lett. 120 (2018) no. 4, 045301, arXiv:1704.05482 [cond-mat.stat-mech].

[28] Z. Bajnok and R. A. Janik, "Classical limit of diagonal form factors and HHL correlators," Journal of High Energy Physics 2017 (2017) no. 1, arXiv: 1607.02830 [hep-th]. http://dx.doi.org/10.1007/JHEP01(2017)063.

[29] M. Takahashi, Thermodynamics of One-Dimensional Solvable Models. Cambridge University Press, 1999.

[30] A. N. Kirillov and V. E. Korepin, "Norms of bound states," Journal of Soviet Mathematics 40 (1988) no. 1, 13-21.

[31] E. Ilievski, J. De Nardis, B. Wouters, J.-S. Caux, F. H. L. Essler, and T. Prosen, "Complete Generalized Gibbs Ensembles in an Interacting Theory," Phys. Rev. Lett. 115 (2015) no. 15, 157201, arXiv:1507.02993 [quant-ph].

[32] M. Mestyán, B. Pozsgay, G. Takács, and M. A. Werner, "Quenching the XXZ spin chain: quench action approach versus generalized Gibbs ensemble," J. Stat. Mech. 4 (2015) 1, arXiv:1412.4787 [cond-mat.stat-mech].

[33] B. Pozsgay and G. Takacs, "Form factors in finite volume I: form factor bootstrap and truncated conformal space," Nucl. Phys. B788 (2008) 167-208, arXiv:0706.1445 [hep-th].

[34] B. Pozsgay and G. Takacs, "Form factors in finite volume II:disconnected terms and finite temperature correlators," Nucl. Phys. B788 (2008) 209-251, arXiv:0706.3605 [hep-th].

[35] L. Hollo, Y. Jiang, and A. Petrovskii, "Diagonal form factors and heavy-heavy-light three-point functions at weak coupling," J. High Energy Phys. 9 (2015) 125, arXiv: 1504.07133 [hep-th].

[36] A. Hutsalyuk, B. Pozsgay, and L. Pristyák, "The LeClair-Mussardo series and nested Bethe Ansatz," Nuclear Physics B 964 (2021) 115306, arXiv:2009.13203 [cond-mat.stat-mech].

[37] L. D. Faddeev, "How Algebraic Bethe Ansatz works for integrable model," arXiv e-prints (1996), arXiv:hep-th/9605187 [hep-th].

[38] R. J. Baxter, Exactly solved models in statistical mechanics. London: Academic Press Inc, 1982.

[39] F. H. L. Essler, H. Frahm, F. Göhmann, A. Klümper, and V. E. Korepin, Th One-Dimensional Hubbard Mode. Cambridge University Press, Cambridge, 2005.

[40] M. Lüscher, "Dynamical charges in the quantized renormalized massive Thirring model," Nucl. Phys. B 117 (1976) no. 2, 475-492. 
[41] E. Ilievski, M. Medenjak, and T. Prosen, "Quasilocal Conserved Operators in the Isotropic Heisenberg Spin-1 /2 Chain," Phys. Rev. Lett. 115 (2015) no. 12, 120601, arXiv:1506.05049 [cond-mat.stat-mech].

[42] E. Ilievski, M. Medenjak, T. Prosen, and L. Zadnik, "Quasilocal charges in integrable lattice systems," J. Stat. Mech. 6 (2016) 064008, arXiv:1603.00440 [cond-mat.stat-mech].

[43] G. Z. Fehér and B. Pozsgay, "Generalized Gibbs Ensemble and string-charge relations in nested Bethe Ansatz," SciPost Phys. 8 (2020) 34, arXiv:1909.04470 [cond-mat.stat-mech].

[44] H. E. Boos, F. Göhmann, A. Klümper, and J. Suzuki, "Factorization of the finite temperature correlation functions of the XXZ chain in a magnetic field," J. Phys. A 40 (2007) 10699, arXiv:0705.2716.

[45] N. Beisert, "The Dilatation operator of $\mathrm{N}=4$ super Yang-Mills theory and integrability," Phys. Rept. 405 (2004) 1-202, arXiv: hep-th/0407277 [hep-th].

[46] T. Bargheer, N. Beisert, and F. Loebbert, "LETTER: Boosting nearest-neighbour to long-range integrable spin chains," J. Stat. Mech. 2008 (2008) no. 11, L11001, arXiv:0807.5081 [hep-th]].

[47] T. Bargheer, N. Beisert, and F. Loebbert, "Long-range deformations for integrable spin chains," J. Phys. A 42 (2009) no. 28, 285205, arXiv:0902.0956 [hep-th].

[48] F. C. Alcaraz and W. F. Wreszinski, "The HeisenbergXXZ Hamiltonian with Dzyaloshinsky-Moriya interactions," Journal of Statistical Physics 58 (1990) no. 1, 45-56. https://doi.org/10.1007/BF01020284

[49] J. Durnin, M. Bhaseen, and B. Doyon, "Non-Equilibrium Dynamics and Weakly Broken Integrability," arXiv e-prints (2020), arXiv:2004.11030 [cond-mat.stat-mech].

[50] D. Szász-Schagrin, B. Pozsgay, and G. Takács, "Weak integrability breaking and level spacing distribution," arXiv e-prints (2021), arXiv:2103.06308 [cond-mat.stat-mech].

[51] R. Modak, S. Mukerjee, and S. Ramaswamy, "Universal power law in crossover from integrability to quantum chaos," Phys. Rev. B 90 (2014) no. 7, 075152, arXiv:1309.1865 [cond-mat.str-el].

[52] H. Boos, M. Jimbo, T. Miwa, F. Smirnov, and Y. Takeyama, "Hidden Grassmann Structure in the XXZ Model," Comm. Math. Phys. 272 (2007) 263-281, hep-th/0606280.

[53] H. Boos, M. Jimbo, T. Miwa, F. Smirnov, and Y. Takeyama, "Hidden Grassmann Structure in the XXZ Model II: Creation Operators," Comm. Math. Phys. 286 (2009)

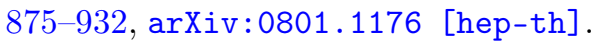

[54] M. Jimbo, T. Miwa, and F. Smirnov, "Hidden Grassmann structure in the XXZ model III: introducing the Matsubara direction," J. Phys. A 42 (2009) no. 30, 304018, arXiv:0811.0439 [math-ph].

[55] H. Boos, M. Jimbo, T. Miwa, F. Smirnov, and Y. Takeyama, "A recursion formula for the correlation functions of an inhomogeneous XXX model," St. Petersburg Math. J. 17 (2006) 85, hep-th/0405044.

[56] H. Boos, M. Jimbo, T. Miwa, F. Smirnov, and Y. Takeyama, "Density Matrix of a Finite Sub-chain of the Heisenberg Anti-ferromagnet," Letters in Mathematica Physics 75 (2006) 201-208, hep-th/0506171.

[57] H. Boos, M. Jimbo, T. Miwa, F. Smirnov, and Y. Takeyama, "Algebraic Representation of Correlation Functions in Integrable Spin Chains," Annales Henr Poincaré 7 (2006) no. 7, 1395-1428, hep-th/0601132. 
[58] H. E. Boos, F. Göhmann, A. Klümper, and J. Suzuki, "Factorization of multiple integrals representing the density matrix of a finite segment of the Heisenberg spin chain," J. Stat. Mech. 2006 (2006) no. 04, P04001, hep-th/0603064.

[59] J. Sato, B. Aufgebauer, H. Boos, F. Göhmann, A. Klümper, M. Takahashi, and C. Trippe, "Computation of Static Heisenberg-Chain Correlators: Control over Length and Temperature Dependence," Phys. Rev. Lett. 106 (2011) no. 25, 257201, arXiv:1105.4447 [cond-mat.str-el].

[60] H. E. Boos and V. E. Korepin, "Quantum spin chains and Riemann zeta function with odd arguments," J. Phys. A 34 (2001) no. 26, 5311, hep-th/0104008.

[61] H. E. Boos, V. E. Korepin, and F. A. Smirnov, "Emptiness formation probability and quantum Knizhnik-Zamolodchikov equation," Nucl. Phys. B 658 (2003) 417-439, hep-th/0209246.

[62] M. Mestyán and B. Pozsgay, "Short distance correlators in the XXZ spin chain for arbitrary string distributions," J. Stat. Mech. 9 (2014) 20, arXiv:1405.0232 [cond-mat.stat-mech].

[63] B. Pozsgay, "Excited state correlations of the finite Heisenberg chain," J. Phys. A 50 (2017) no. 7, 074006, arXiv:1605.09347 [cond-mat.stat-mech].

[64] H. Boos, M. Jimbo, T. Miwa, F. Smirnov, and Y. Takeyama, "Traces on the Sklyanin algebra and correlation functions of the eight-vertex model," J. Phys. A 38 (2005) no. 35, 7629-7659, arXiv: hep-th/0504072 [hep-th].

[65] F. Göhmann and V. E. Korepin, "Solution of the quantum inverse problem," J. Phys. A 33 (2000) no. 6, 1199, arXiv: hep-th/9910253.

[66] J. M. Maillet and V. Terras, "On the quantum inverse scattering problem," Nucl. Phys. B 575 (2000) 627-644, hep-th/9911030.

[67] B. Doyon, "Exact large-scale correlations in integrable systems out of equilibrium," SciPost Phys. 5 (2018) 054, arXiv:1711.04568 [math-ph].

[68] A. Cortés Cubero and M. Panfil, "Generalized hydrodynamics regime from the thermodynamic bootstrap program," SciPost Phys. 8 (2020) no. 1, 004 , arXiv:1909.08393 [cond-mat.stat-mech].

[69] F. Göhmann, K. K. Kozlowski, and J. Suzuki, "High-temperature analysis of the transverse dynamical two-point correlation function of the XX quantum-spin chain," arXiv e-prints (2019), arXiv:1905.04922 [math-ph].

[70] F. Göhmann, K. K. Kozlowski, J. Sirker, and J. Suzuki, "The equilibrium dynamics of the XX chain revisited," arXiv e-prints (2019), arXiv:1906.03143 [cond-mat.stat-mech].

[71] F. Göhmann, K. K. Kozlowski, and J. Suzuki, "Long-time large-distance asymptotics of the transverse correlation functions of the XX chain in the spacelike regime," Letters in Mathematical Physics 110 (2020) no. 7, 1783-1797, arXiv: 1908.11555 [cond-mat.stat-mech].

[72] C. Babenko, F. Göhmann, K. K. Kozlowski, and J. Suzuki, "A thermal form factor series for the longitudinal two-point function of the Heisenberg-Ising chain in the antiferromagnetic massive regime," arXiv e-prints (2020), arXiv:2011.12752 [cond-mat.stat-mech].

[73] C. Babenko, F. Göhmann, K. K. Kozlowski, J. Sirker, and J. Suzuki, "Exact real-time longitudinal correlation functions of the massive XXZ chain," arXiv e-prints (2020), arXiv:2012.07378 [cond-mat.stat-mech].

[74] B. Doyon, "Hydrodynamic projections and the emergence of linearised Euler equations in one-dimensional isolated systems," arXiv e-prints (2020), arXiv:2011.00611 [math-ph]. 
[75] E. Ilievski and J. De Nardis, "Ballistic transport in the one-dimensional Hubbard model: The hydrodynamic approach," Phys. Rev. B 96 (2017) no. 8, 081118, arXiv:1706.05931 [cond-mat.stat-mech].

[76] Y. Nozawa and H. Tsunetsugu, "Generalized hydrodynamic approach to charge and energy currents in the one-dimensional Hubbard model," Phys. Rev. B 101 (2020) no. 3, 035121, arXiv:1910.02427 [cond-mat.stat-mech]].

[77] Y. Nozawa and H. Tsunetsugu, "Generalized hydrodynamics study of the one-dimensional Hubbard model: Stationary clogging and proportionality of spin, charge, and energy currents," Phys. Rev. B 103 (2021) no. 3, 035130, arXiv:2008.06522 [cond-mat.stat-mech].

[78] B. Pozsgay, Y. Jiang, and G. Takács, "TT̄ -deformation and long range spin chains," JHEP 03 (2020) 092, arXiv: 1911.11118 [hep-th].

[79] E. Marchetto, A. Sfondrini, and Z. Yang, " $T \bar{T}$ deformations and integrable spin chains," Phys. Rev. Lett. 124 (2020) no. 10, 100601, arXiv:1911.12315 [hep-th].

[80] G. A. P. Ribeiro and A. Klümper, "Correlation functions of the integrable $\$ S U(n) \$$ spin chain," J. Stat. Mech. 1 (2019) 013103, arXiv:1804.10169 [math-ph].

[81] H. Boos, A. Hutsalyuk, and K. S. Nirov, "On the calculation of the correlation functions of the $\mathfrak{s l}_{3}$-model by means of the reduced qKZ equation," J. Phys. A 51 (2018) no. 44, 445202, arXiv: 1804.09756 [hep-th].

[82] D. Martin and F. Smirnov, "Problems with Using Separated Variables for Computing Expectation Values for Higher Ranks," Letters in Mathematical Physics 106 (2016) no. 4, 469-484, arXiv:1506.08042 [math-ph]. 